\title{
Hyphal Development in Candida albicans Requires Two Temporally Linked Changes in Promoter Chromatin for Initiation and Maintenance
}

\author{
Yang Lu, Chang Su, Allen Wang, Haoping Liu* \\ Department of Biological Chemistry, University of California, Irvine, California, United States of America
}

\begin{abstract}
Phenotypic plasticity is common in development. For Candida albicans, the most common cause of invasive fungal infections in humans, morphological plasticity is its defining feature and is critical for its pathogenesis. Unlike other fungal pathogens that exist primarily in either yeast or hyphal forms, C. albicans is able to switch reversibly between yeast and hyphal growth forms in response to environmental cues. Although many regulators have been found involved in hyphal development, the mechanisms of regulating hyphal development and plasticity of dimorphism remain unclear. Here we show that hyphal development involves two sequential regulations of the promoter chromatin of hypha-specific genes. Initiation requires a rapid but temporary disappearance of the Nrg1 transcriptional repressor of hyphal morphogenesis via activation of the CAMP-PKA pathway. Maintenance requires promoter recruitment of Hda1 histone deacetylase under reduced Tor 1 (target of rapamycin) signaling. Hda1 deacetylates a subunit of the NuA4 histone acetyltransferase module, leading to eviction of the NuA4 acetyltransferase module and blockage of Nrg1 access to promoters of hypha-specific genes. Promoter recruitment of Hda1 for hyphal maintenance happens only during the period when Nrg1 is gone. The sequential regulation of hyphal development by the activation of the CAMP-PKA pathway and reduced Tor1 signaling provides a molecular mechanism for plasticity of dimorphism and how C. albicans adapts to the varied host environments in pathogenesis. Such temporally linked regulation of promoter chromatin by different signaling pathways provides a unique mechanism for integrating multiple signals during development and cell fate specification.
\end{abstract}

Citation: Lu Y, Su C, Wang A, Liu H (2011) Hyphal Development in Candida albicans Requires Two Temporally Linked Changes in Promoter Chromatin for Initiation and Maintenance. PLoS Biol 9(7): e1001105. doi:10.1371/journal.pbio.1001105

Academic Editor: Joseph Heitman, Duke University Medical Center, United States of America

Received December 7, 2010; Accepted May 31, 2011; Published July 19, 2011

Copyright: (c) 2011 Lu et al. This is an open-access article distributed under the terms of the Creative Commons Attribution License, which permits unrestricted use, distribution, and reproduction in any medium, provided the original author and source are credited.

Funding: This work was supported by the US National Institutes of Health grant R01GM/AI55155 to HL. HL was also supported in part by the NIH National Institute of General Medical Sciences Center grant GM76516. The funders had no role in study design, data collection and analysis, decision to publish, or preparation of the manuscript.

Competing Interests: The authors have declared that no competing interests exist.

Abbreviations: ChIP, chromatin immunoprecipitation; HAT, histone acetyltransferase; HDAC, histone deacetylase; PKA, protein kinase A; TOR, target of rapamycin

*E-mail: H4liu@uci.edu

\section{Introduction}

Many organisms or cells are able to alter their phenotype or developmental fate in response to changes in their environment, a phenomenon referred to as plasticity. Plasticity is seen in a broad range of biological systems from embryo development to memory formation where long-lasting effects on gene expression outlive an initial transient signal. The dynamic process of cell fate specification is determined by a network of regulatory genes. The architecture of the network defines the temporal order of specification events. Therefore, understanding temporal dynamic regulation of gene expression in response to extracellular signals is critical for our comprehension of cell fate specification and plasticity.

Morphological plasticity is a defining feature of Candida albicans, a major opportunistic fungal pathogen of humans [1]. C. albicans resides as harmless commensal flora in the gastrointestinal tract and mucosal membranes of healthy individuals, but when the host immune system is suppressed, the fungus can disseminate and cause systemic infections. Unlike many other pathogenic fungi that exist primarily in either yeast or hyphal forms and infect a specific organ, C. albicans is able to undergo reversible morphological changes between yeast, pseudohyphal, and hyphal forms of growth in response to environmental cues and can successfully infect many different anatomical sites of the human host. Its morphological plasticity is the most important virulence attribute of C. albicans [2]. Hyphae have invasive properties that can promote tissue penetration and escape from immune cells, whereas yeast cells are suited for dissemination in the bloodstream. Several of the genes that are specifically expressed in hyphae encode virulence factors. For example, HWP1, ALS3, and RBT5 encode cell wall proteins that are important for adhesion to host cells and iron acquisition from the host [3-6].

Several signal transduction pathways are involved in the regulation of hyphal development. Among them, the cAMPdependent protein kinase A (PKA) pathway plays an essential role in hyphal morphogenesis and virulence [7]. The adenylate cyclase Cyrl and its associated protein are indispensable for hyphal growth under all conditions [8-10]. Tpk1 and Tpk2 are catalytic subunits of PKA; each plays distinct functions in hyphal development [11-13]. Efgl and Flo8, two transcription regulators essential for hyphal development and virulence [14-16], are 


\section{Author Summary}

Many organisms are able to change their phenotype in response to changes in the environment, a phenomenon referred to as plasticity. Candida albicans, a major opportunistic fungal pathogen of humans, can undergo reversible morphological changes between yeast (spherical) and hyphal (filamentous) forms of growth in response to environmental cues. This morphological plasticity is essential for its pathogenesis and survival in its hosts. In this study, we show that hyphal development is initiated and maintained by two major nutrient-sensing cellular growth pathways that act by removing the inhibition provided by the transcriptional repressor Nrg1. While initiation requires a rapid but temporary disappearance of Nrg1 via activation of the CAMP-dependent protein kinase A pathway, maintenance requires the recruitment to promoters of the Hda1 histone deacetylase under conditions of reduced signaling by the target of rapamycin (TOR) kinase, leading to chromatin remodeling that blocks Nrg1 access to the promoters of hypha-specific genes. We observed that recruitment of Hda1 to promoters happens only during the time window when Nrg1 is absent. Such temporally linked regulation of promoter chromatin by different signaling pathways provides a unique mechanism for integrating multiple signals in the regulation of gene expression and phenotypic plasticity during development and cell fate specification.

implicated to function downstream of the cAMP-PKA pathway $[17,18]$. The hyphal transcriptional program is repressed by Tup 1 through sequence-specific DNA-binding proteins [19-27], of which Nrgl plays a major role. $n r g 1$ mutant cells are constitutively filamentous under all conditions, similar to tup1 cells $[25,26]$. Ectopic expression of $\mathcal{N R G 1}$ inhibits hyphal filamentation in all growth conditions $[28,29]$. Although molecular genetic analyses have identified a number of key transcriptional regulators of hyphal morphogenesis, our understanding of the transcriptional regulation that governs the yeast-to-hypha transition remains rudimentary.

The yeast-to-hypha transition can be induced by a wide range of media and environmental conditions in vitro [1]. Serum in combination with a rise in temperature to $37^{\circ} \mathrm{C}$ gives the most robust induction of hyphae. Simple inoculation of stationary cells into fresh medium at $37^{\circ} \mathrm{C}$ is also a powerful but transient inducer of hyphae [30]. Several induction signals are transmitted through Cyrl, including $\mathrm{CO}_{2} / \mathrm{HCO}_{3}{ }^{-}$and peptidoglycan found in serum, as well as a rise in temperature [31-33]. Cyrl is also regulated by Ras1, Ras2, and Gpa2 in response to nutrients [34-38]. Farnesol, a quorum-sensing molecule secreted to the medium by $C$. albicans cells as a cell density signal [39], exerts its inhibitory effects on germ-tube formation through Ras1-Cyrl [40]. However, many widely used hyphal-inducing media are poor in nitrogen and carbon sources that are not favorable for the activation of the cAMP-PKA pathway, raising the question of how C. albicans can undergo hyphal development in both rich and poor media. The target of rapamycin (TOR) protein kinase pathway is another major nutrient-sensing pathway conserved in C. albicans [41]. Rapamycin can both inhibit hyphal formation on solid medium and promote cell aggregation in liquid medium in C. albicans $[41,42]$, but it does not induce yeast-to-hyphal development.

Transcriptional activation or repression in response to external stimuli is often mediated through dynamic changes in promoter chromatin structure. This regulation occurs at many different levels, including posttranslational modification of histones [43], chromatin remodeling [44], incorporation of histone variants [45], and cotranscriptional chromatin disassembly and assembly $[46,47]$. All of these dynamic processes work in concert to establish or alter the local properties of chromatin, although the relative importance and order of these processes vary at each individual promoter. Histone acetyltransferases (HATs) and deacetylases (HDACs) play important roles in regulating chromatin structure and transcription [48]. Histone acetylation is a dynamic reversible process, and the balance of histone acetylation is important for proper cellular functions [49]. We have previously shown a dynamic nucleosomal $\mathrm{H} 4$ acetylation at the promoters of hypha-specific genes during hyphal induction [50]. H4 histone acetyltransferase complex NuA4 is recruited to promoters in both yeast and hyphal forms and is required for the induction of hyphal genes [50]. Here we show that the dynamic increase and decrease in $\mathrm{H} 4$ acetylation at the promoters during hyphal induction correlates with the sequential dissociation and association of two different HDAGs, Rpd3 and Hdal, respectively. Concomitant with the dissociation of Rpd3 upon hyphal initiation, $\mathrm{Nrg} 1$ protein disappears from cells in response to the activation of the cAMPPKA pathway, a step essential for hyphal initiation. Although Nrgl protein returns after hyphal initiation, promoter recruitment of Hdal under reduced Tor 1 signaling leads to deacetylation and eviction of the NuA4 HAT module, nucleosome reassembly, and inhibition of $\mathrm{Nrg} 1$ access to the promoters of hypha-specific genes, a step essential for sustained hyphal development. Importantly, disappearance of $\mathrm{Nrg} 1$ during hyphal initiation is a prerequisite for promoter recruitment of Hdal. The sequential regulation of hyphal development by the activation of the cAMP-PKA pathway and reduced Torl signaling provides a molecular mechanism for the plasticity of dimorphism and how C. albicans adapts to the varied host environments in pathogenesis.

\section{Results}

Yeast-to-Hyphal Development Involves Two Temporally Related Phases of Removing Nrg1 Inhibition

The transcription factor $\mathrm{Nrgl}$ plays an essential role in repressing hyphal development [22,25,26,51]. The significance of $\mathrm{Nrgl}$ is underscored by recent phenotypic profiling of 143 transcriptional regulator knockout mutants, where only $n r g 1$ and tup1 mutants are filamentous under all conditions examined [52]. Relief of the transcriptional repression by Nrg1-Tupl may be the essential and regulated step for the activation of the hyphal transcriptional program under all conditions. Indeed, NRG1 transcript level has been shown to be reduced in hyphae $[22,25,26,53]$. However, how $\mathrm{Nrg} 1$ is regulated at the protein level and whether $\mathrm{Nrgl}$ directly binds to the promoters of hyphaspecific genes is not clear. By chromatin immunoprecipitation (ChIP) of C-terminal Myc-tagged Nrgl, we found that Nrgl was at the promoters of hypha-specific genes HWP1, ALS3, and ECE1 during yeast growth (Figure 1). We next analyzed the levels of promoter-bound $\mathrm{Nrgl}$ during yeast to hyphal morphological development under four related conditions in YPD medium that induce three distinctive developmental responses (Figure 1A). Cells inoculated to YPD with or without serum at $30^{\circ} \mathrm{C}$ remain in the yeast growth form. Cells inoculated into YPD at $37^{\circ} \mathrm{C}$ in the absence of serum show normal germ tube formation, but are unable to maintain hyphal development. Serum, in combination with a shift in temperature to $37^{\circ} \mathrm{C}$, induces robust and sustained hyphal growth. By a time course ChIP, we found that, upon hyphal induction, $\mathrm{Nrg} 1$ dissociated rapidly from the promoters and remained at low levels during hyphal elongation in YPD with serum (Figure 1B). In contrast, levels of promoter-associated Nrgl 
A
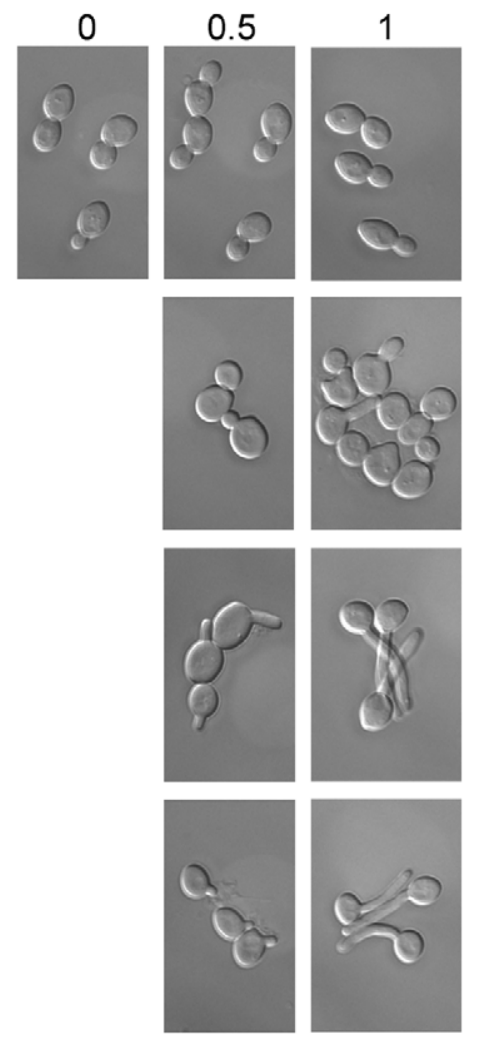

B
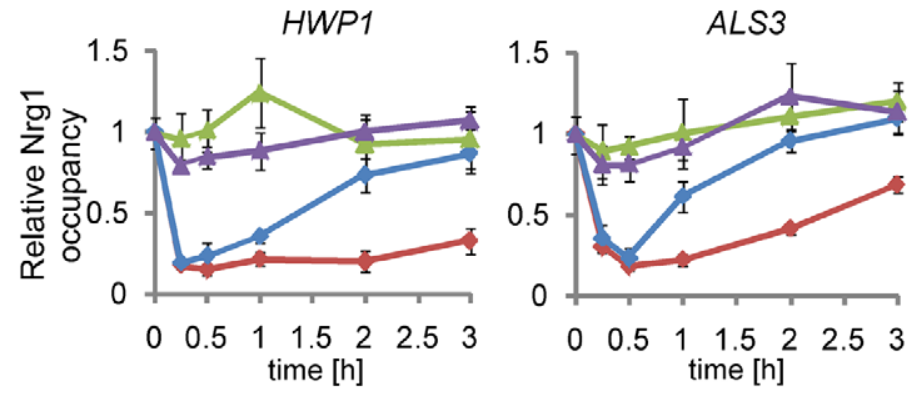

C

\begin{tabular}{|c|c|c|c|c|c|c|}
\hline \multicolumn{6}{|c|}{ YPD $30^{\circ} \mathrm{C}$} & \multirow{3}{*}{ Nrg1-Myc } \\
\hline 0 & 0.25 & \multirow{2}{*}{0.0} & \multirow{2}{*}{1} & \multirow{2}{*}{2} & \multirow{2}{*}{$3(\mathrm{~h})$} & \\
\hline-1 & & & & & & \\
\hline E & $=$ & & & $=$ & 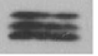 & Cdc28 \\
\hline & & YPD & $7^{\circ} \mathrm{C}$ & & & \\
\hline 0 & 0.25 & 0.5 & 1 & 2 & $3(h)$ & \\
\hline 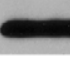 & & & 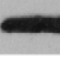 & 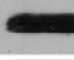 & $\longrightarrow$ & Nrg1-Myc \\
\hline & & & & $F$ & $E$ & $\mathrm{Cdc} 28$ \\
\hline
\end{tabular}

3
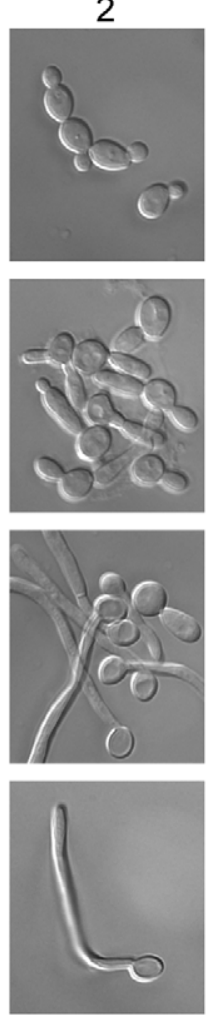

5 (h)
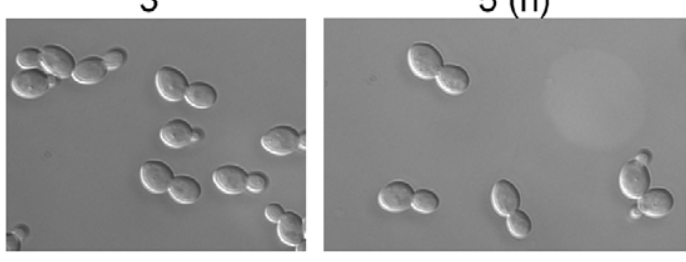

YPD $30^{\circ} \mathrm{C}$
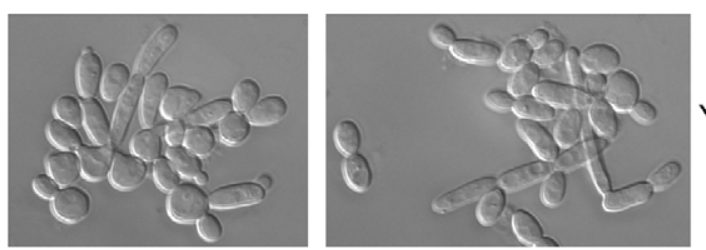

YPD+Serum $30^{\circ} \mathrm{C}$
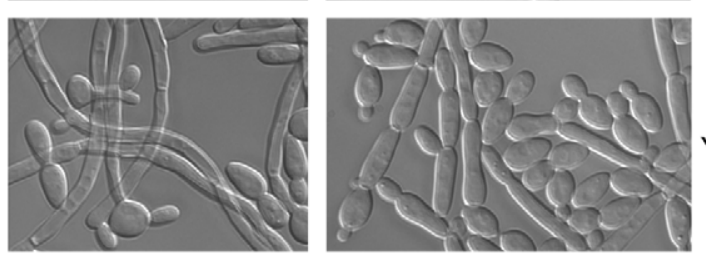

YPD $37^{\circ} \mathrm{C}$

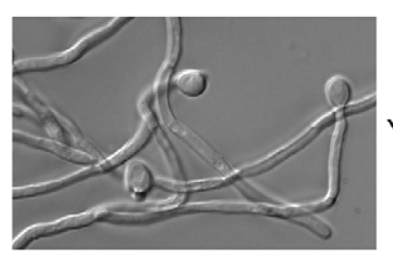

YPD+Serum $37^{\circ} \mathrm{C}$

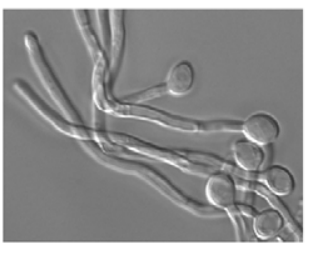

ECE1

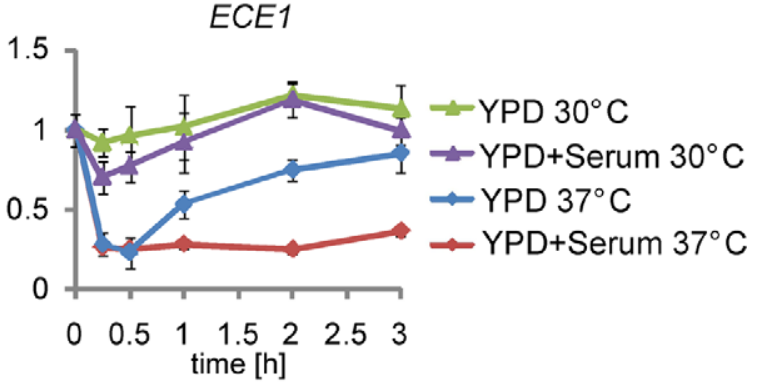

YPD+Serum $30^{\circ} \mathrm{C}$

\begin{tabular}{llllll}
\hline 0 & 0.25 & 0.5 & 1 & 2 & $3(\mathrm{~h})$ \\
& $=$ & & & & \\
Nrg1-Myc
\end{tabular}

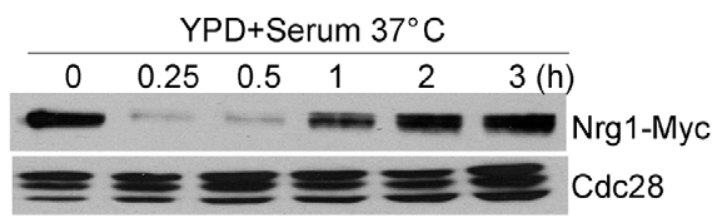

Figure 1. Initiation and maintenance of hyphal development by removing Nrg1 inhibition. An overnight culture of wild-type cells carrying Nrg1-Myc (HLY3922) were diluted 1:50 into the indicated medium at $30^{\circ} \mathrm{C}$ or $37^{\circ} \mathrm{C}$ in the presence or absence of $10 \%$ serum, and cells were collected at $0 \mathrm{~min}, 15 \mathrm{~min}, 30 \mathrm{~min}, 1 \mathrm{~h}, 2 \mathrm{~h}, 3 \mathrm{~h}$, and $5 \mathrm{~h}$ for cell morphology (A), ChIP (B), and Western blot (C) experiments. ChIP DNA were quantitated by qPCR with primers at the UAS regions of HWP1, ALS3, and ECE1, as described [50]. The ADE2 coding region was used as a control. The Nrg1-Myc enrichment is presented as a ratio of HWP1, ALS3, or ECE1 IP (bound/input) versus the control IP (bound/input). The $0 \mathrm{~h}$ values on HWP1 were set to 1.00. The ChIP data show the average of three independent QPCR data with error bars representing the SEM. Western analysis was carried out using a peroxidase-conjugated anti-c-myc (Roche Diagnostics) antibody to assess levels of Nrg1-myc, and with an anti-PSTAIRE (Cdc28, Santa Cruz) antibody for loading control. doi:10.1371/journal.pbio.1001105.g001 
recovered after the initial decrease under the condition of YPD without serum, which correlated with the conversion to the yeast growth phase around $3 \mathrm{~h}$ after the induction (Figure 1A and 1B). Unexpectedly, Western analyses indicated that Nrgl protein levels decreased sharply at 15 and $30 \mathrm{~min}$ upon hyphal induction at $37^{\circ} \mathrm{C}$ coinciding with germ tube formation. However, the Nrgl level recovered after $1 \mathrm{~h}$ of hyphal induction (Figure $1 \mathrm{C}$ ). Serum was not required for the disappearance of $\mathrm{Nrgl}$, but was critical for sustained hyphal development and excluding Nrgl from promoters during hyphal elongation (Figure 1). The Western analysis of cells after $3 \mathrm{~h}$ of hyphal induction could not discriminate $\mathrm{Nrg} 1$ protein from apical cells and subapical cells. It is possible that $\mathrm{Nrg} 1$ protein was absent in the apical cell of each hypha and, therefore, allowed sustained hyphal growth from the apical cells. We determined Nrgl localization by immunofluorescence in hyphal cells after $5 \mathrm{~h}$ of growth in YPD with serum at $37^{\circ} \mathrm{C}$ and found $\mathrm{Nrgl}$ in the nucleus of both apical and subapical cells (Figure S1), excluding this possibility. Our data suggest that hyphal development involves two phases of releasing Nrgl inhibition: the first for initiation and the second for maintenance. We suggest that initiation requires a transient down-regulation of $\mathrm{Nrg} 1$ protein level, while maintenance requires a regulation that prevents $\mathrm{Nrgl}$ from binding at the promoters of hypha-specific genes.

\section{The cAMP-PKA Pathway Is Required for the Down-Regulation of Nrg1 for Hyphal Initiation}

Several lines of evidence suggest that the rapid and temporary disappearance of $\mathrm{Nrg} 1$ protein upon hyphal induction is regulated by the cAMP-PKA pathway. First, we found that Nrgl disappearance required the cAMP-PKA pathway as Nrgl protein level did not show an obvious reduction in $c y r 1$ and $t p k 2$ mutants (Figure 2A). Similarly, no obvious change in Nrgl level was observed in an efg 1 or flo 8 mutant (Figure 2A). Second, the shift to $37^{\circ} \mathrm{C}$ during hyphal induction was critical for the observed $\mathrm{Nrg} 1$ disappearance (Figure 1C). This is consistent with the report that Hsp90 regulates hyphal development via Cyrl in response to a shift in temperature [33]. Third, the major quorum-sensing molecule farnesol, at a physiological concentration, completely blocked the down-regulation of Nrgl (Figure 2A). Farnesol is reported to exert its inhibitory effects on hyphal initiation through Cyrl $[39,40]$. Consistent with the inhibitory effect of farnesol on the down-regulation of $\mathrm{Nrgl}$, different folds of dilution during inoculation could lead to different durations of $\mathrm{Nrgl}$ downregulation (Figure 1G versus Figure 2A). We also observed that hyphal initiation correlated with $\mathrm{Nrgl}$ disappearance in all liquid media, including nutrient-poor media (Figure 2B). However, hyphal initiation in a poor medium required inoculation of cells from a saturated overnight culture. This result is consistent with the report that release from quorum-sensing molecules, such as farnesol, triggers the yeast-to-hypha transition at $37^{\circ} \mathrm{C}$ independent of the inoculation media [30]. The timing of hyphal initiation was slower in medium with mannitol than that with glucose (Figure 2B), consistent with the activation of the cAMP pathway by glucose in addition to the release of inhibition from farnesol. Together, our observations suggest that hyphal initiation involves a down-regulation of $\mathrm{Nrgl}$ protein in response to the activation of the cAMP-PKA pathway by a combination of temperature shift, release from inhibition by farnesol, and nutrients in rich media.

The NRG1 transcript has been reported to be reduced during hyphal induction in serum containing medium [22,25,26,53]. We found that the decrease in $\mathcal{N} R G 1$ expression during hyphal growth was dependent on the cAMP-PKA pathway, as Cyrl (unpublished data) or Tpk2 was required for the reduction in $\mathcal{N} R G 1$ expression (Figure 2C), consistent with the observation that $\mathcal{N} R G 1$ transcript level was not down-regulated in the efg1 mutant during hyphal induction in serum [26]. The transcriptional down-regulation was essential for the initiation of hyphal development, as ectopic expression of NRG1 under the MAL2 promoter in wild-type cells could not initiate hyphal development even under robust induction conditions (Figure 2D). However, the decrease in NRG1 transcript alone is not sufficient to provide the temporal dynamic change in $\mathrm{Nrg} 1$ protein level during hyphal development. We observed that, by promoter shut-down experiments, $\mathrm{Nrgl}$ was unstable when cells were inoculated into fresh medium at $30^{\circ} \mathrm{C}$ either from an overnight culture (Figure 2E) or a logarithmic growing culture (unpublished data). Adding farnesol inhibited $\mathrm{Nrg} 1$ degradation, suggesting that release from farnesol inhibition during inoculation is important in triggering the degradation of $\mathrm{Nrg}$ 1. Therefore, the rapid and temporary disappearance of $\mathrm{Nrgl}$ protein is the combined result of a cAMP-PKA dependent downregulation of $\mathcal{N} R G 1$ expression and a burst of $\mathrm{Nrgl}$ degradation upon release from farnesol during inoculation. This temporary removal of $\mathrm{Nrgl}$ is essential for hyphal induction.

\section{Promoter-Recruitment of Hda1 Is Required for Sustained Hyphal Development by Limiting Promoter Access to Nrg1 \\ One possible mechanism for the reduced promoter access by} Nrgl during hyphal elongation is a change in promoter chromatin. C. albicans has one class II HDAC Hdal that functions as a repressor for phenotypic switching [54]. We found that cells deleted of HDA1 were unable to maintain hyphal growth. They were impaired in sustained hyphal development and transcription of hypha-specific genes, but had no detectable defects during initial germ-tube formation (Figures $3 \mathrm{AB}, \mathrm{S} 2 \mathrm{~A}$ and Table S1A). Furthermore, levels of promoter-bound $\mathrm{Nrgl}$ increased dramatically in the hdal mutant after hyphal initiation, suggesting that Hdal is required to prevent $\mathrm{Nrgl}$ binding to the promoters for sustained hyphal growth (Figures 3C and S2B). These observations of the hda1 mutant are similar to that of wild-type cells inoculated into rich medium at $37^{\circ} \mathrm{C}$ without serum (Figure 1). To determine whether Hdal functions directly on the promoters of hyphaspecific genes, we performed a time course ChIP of Hdal. We found that Hdal was recruited to the promoters during hyphal induction in a serum-dependent manner (Figures 3D and S2C). We suggest that serum-induced $\mathrm{Hdal}$ recruitment to the promoters of hypha-specific genes leads to a change in promoter chromatin that is no longer accessible to $\mathrm{Nrg} 1$, leading to sustained hyphal development.

In contrast to Hdal, we found that the Rpd3 HDAG preferentially associated with hypha-specific promoters in yeast cells and dissociated rapidly from the promoters upon hyphal induction, similar to the dynamic dissociation of $\mathrm{Nrg} 1$ (Figure S3). The sequential dissociation and recruitment of two different HDACs to the hypha-specific promoters likely account for the dynamic change in nucleosomal $\mathrm{H} 4$ acetylation levels during hyphal induction with the peak $\mathrm{H} 4$ acetylation at 30 min [50].

\section{The Promoter-Associated Hda1 Deacetylates the Yng2 Subunit of NuA4 Histone Acetyltransferase (HAT) module, Leading to the Reduction of Yng2 and NuA4 HAT at the Promoters of Hypha-Specific Genes}

How does promoter-associated Hdal prevent $\mathrm{Nrgl}$ from binding to the promoters of hypha-specific genes during hyphal elongation? One potential mechanism is chromatin remodeling. We have previously shown nucleosome reassembly and a decrease in $\mathrm{H} 4$ acetylation at the UAS regions of hypha-specific genes 
A

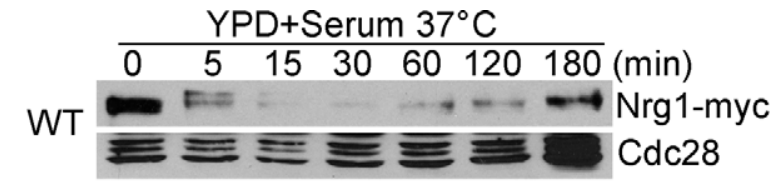

cyr1 $=0 \mathrm{Nrg} 1-\mathrm{myc}$
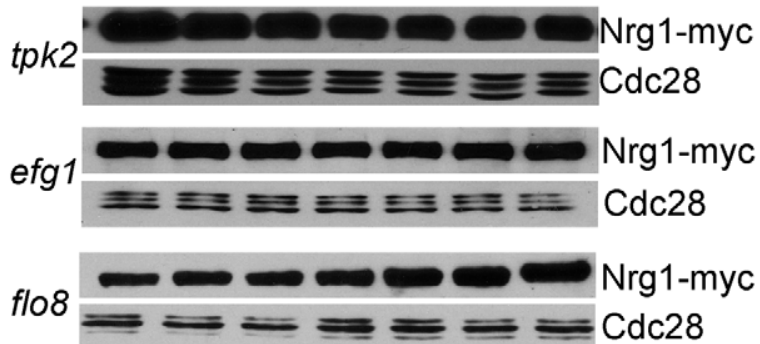

WT

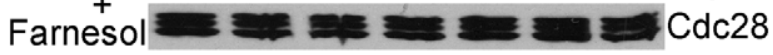

C

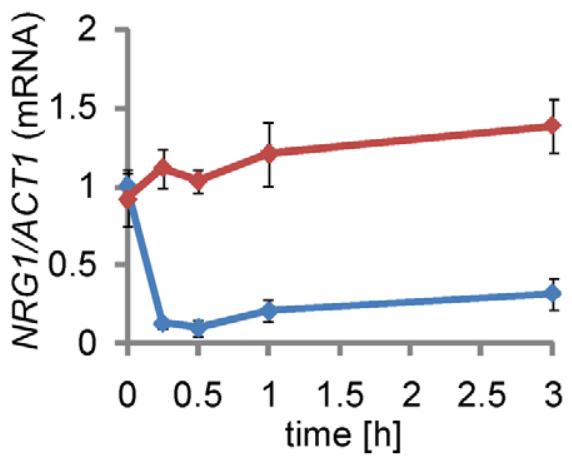

D

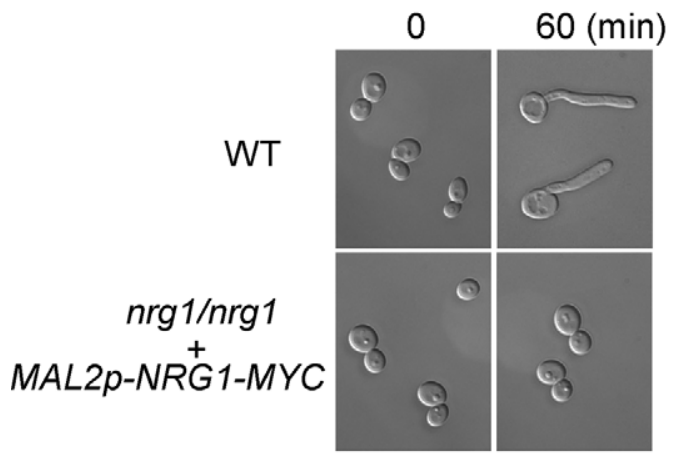

$\rightarrow$ WT $\sim$ tpk2
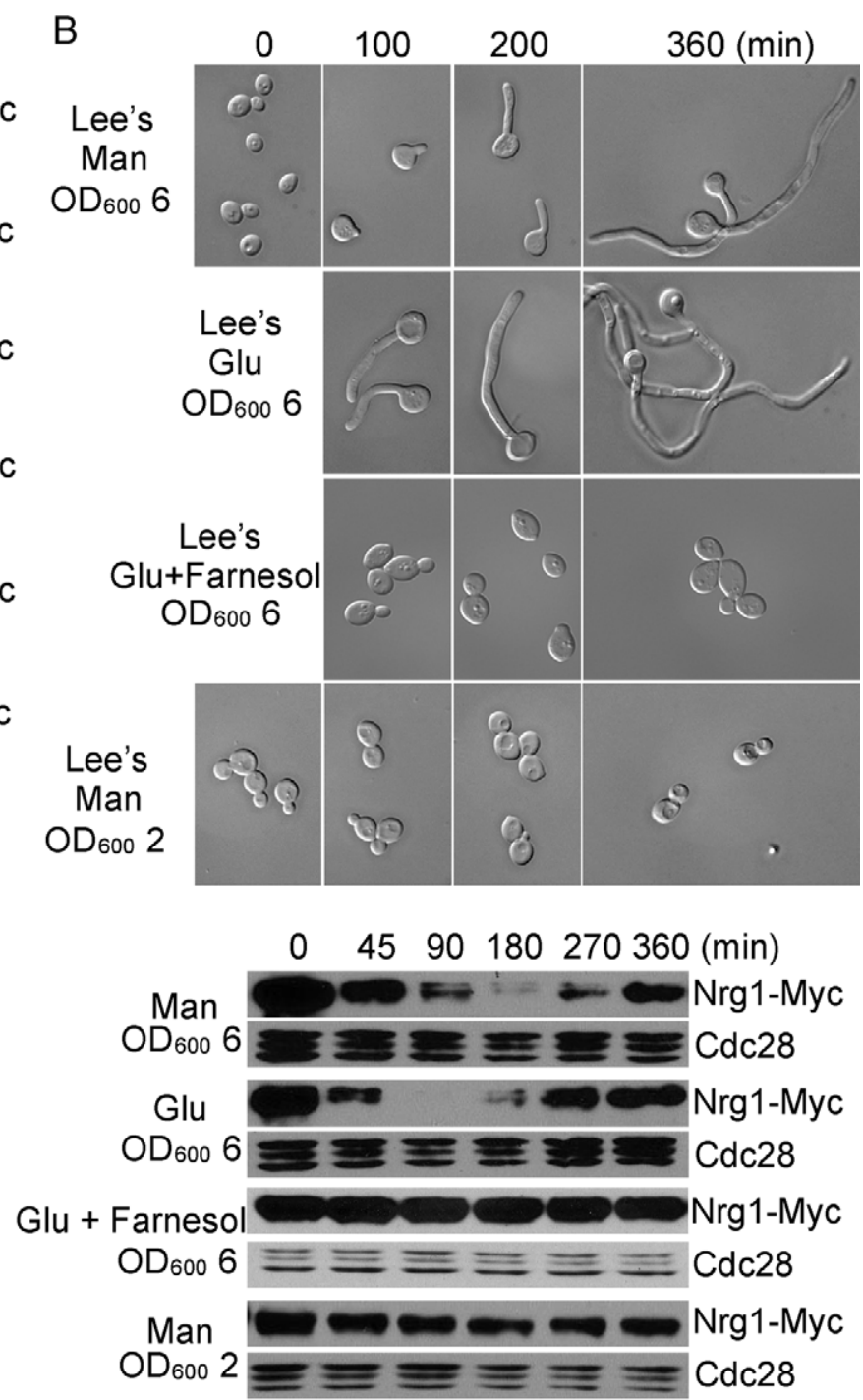

E

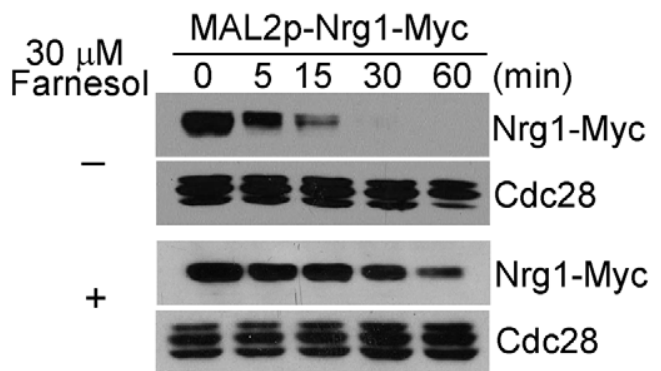

Figure 2. Hyphal initiation requires CAMP-PKA dependent down-regulation of Nrg1 protein. (A) Components of the CAMP-PKA pathway and release from farnesol inhibition are required for down-regulation of Nrg1 protein during hyphal initiation. Western analysis of Nrg1-Myc during hyphal induction in wild-type, cyr1, tpk2, efg1, and flo8 mutants. Cells of wild type and mutants carrying Nrg1-Myc were diluted 1:100 into fresh YPD $+10 \%$ serum medium at $37^{\circ} \mathrm{C}$ with or without $30 \mu \mathrm{M}$ farnesol as indicated. (B) Cell morphology and Western blot analysis of wild-type cells carrying Nrg1-myc. Cells from overnight cultures of the indicated $\mathrm{OD}_{600}$ were diluted 1:100 into Lee's media with either mannitol or glucose. Adding $30 \mu \mathrm{M}$ farnesol into Lee's glucose medium blocked Nrg1 down-regulation and hyphal development. (C) Tpk2 is essential for transcriptional downregulation of NRG1 during hyphal induction. NRG1 mRNA levels were determined by qRT-PCR in WT and tpk2/tpk2 strains over a time course of hyphal induction (YPD+10\% Serum). The signal obtained from ACT1 mRNA was used as a loading control for normalization. The data show the average of three independent QRT-PCR experiments with error bars representing the SEM. (D) Constitutive expression of Nrg1 blocks germ tube formation. Cells of wild-type and $n r g 1 / n r g 1$ carrying Nrg1-Myc under the MAL2 promoter were grown in YPMaltose medium at $30^{\circ} \mathrm{C}$ overnight and were diluted at 1:20 into pre-warmed YPMaltose medium at $37^{\circ} \mathrm{C}$. (E) Nrg1 protein stability by promoter-shutdown. Western or wild-type cells carrying Nrg1-Myc under the MAL2 promoter inoculated from overnight culture into fresh YPD medium at $30^{\circ} \mathrm{C}$ with and without $30 \mu \mathrm{M}$ farnesol. doi:10.1371/journal.pbio.1001105.g002 
A

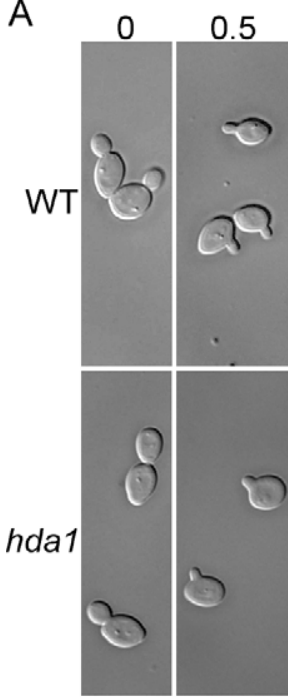

B

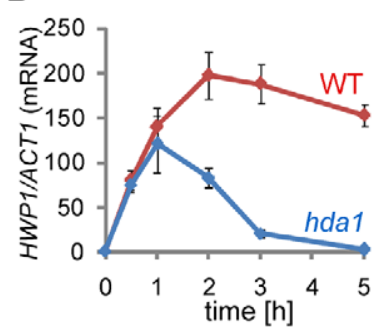

1

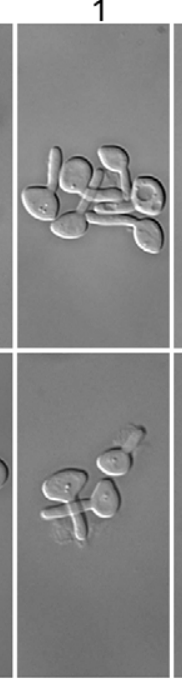

C

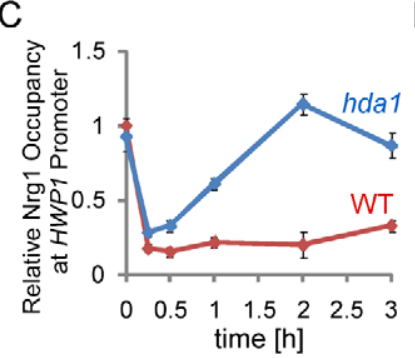

3

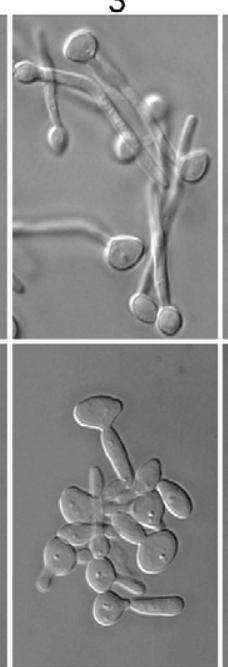

D

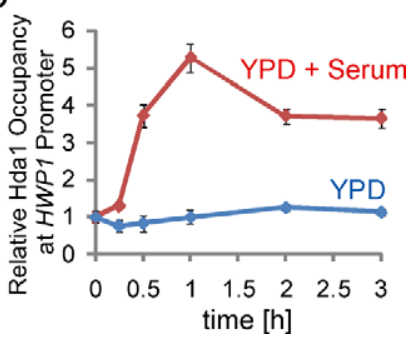

Figure 3. Promoter recruitment of Hda1 is required for hyphal maintenance by inhibiting Nrg1 access to the promoters of hyphaspecific genes. (A, B) Hda1 is required for maintenance of hyphal development as shown by morphology and expression levels of hypha-specific genes during hyphal induction. Cells of wild-type (TS3.3+pBES116) and hda1/hda1 (HLY4032+pBES116) strains were diluted into YPD with 10\% serum at $37^{\circ} \mathrm{C}$. HWP1, ALS3, and ECE1 mRNA levels were determined by qRT-PCR. The signal obtained from ACT1 mRNA was used as a loading control for normalization. (C) Nrg1-Myc binds the promoters of hypha-specific genes in hda1 cells during hyphal elongation in YPD with 10\% serum. (D) Serumdependent binding of Hda1-Myc to the hyphal promoters in wild-type cells during hyphal induction. Cells of wild-type strain containing Hda1-Myc (HLY4033) were diluted into pre-warmed YPD medium at $37^{\circ} \mathrm{C}$ in the presence or absence of $10 \%$ serum. ChIP DNA in (C, D) were quantitated by qPCR with primers at the UAS regions of hypha-specific genes as described in Figure 1. Data for ALS3 and ECE1 from (B, C, D) are shown in Figure S2. All data show the average of three independent qRT-PCR or qPCR experiments with error bars representing the SEM.

doi:10.1371/journal.pbio.1001105.g003

during hyphal elongation [50]. The decrease in H4 acetylation could be a result of $\mathrm{H} 4$ deacetylation by promoter-associated Hdal or eviction of NuA4 from the promoters. S. cerevisiae Hdal is a histone deacetylase specific for H3 and H2B [55]. Therefore, H4 may not be a substrate of the C. albicans Hda1. Yng2, a subunit of the NuA4 histone acetyltransferase HAT complex essential for HAT activity, is acetylated at lysine 170 by $\mathrm{NuA} 4$ and deacetylated by Rpd3 in S. cerevisiae [56]. Deacetylation of Yng2 leads to its degradation and eviction of chromatin-bound Yng2 with Esa1, the catalytic subunit of NuA4. In C. albicans, the NuA4 HAT complex is recruited to the promoters of hypha-specific genes [50]. By immunoprecipitation with anti-acetylated-lysine antibodies, we detected acetylated Yng2 in wild-type cells, but observed a dramatic increase in the level of acetylated Yng2 in the hda1 mutant (Figure 4A). This suggests that deacetylation of Yng2 in vivo depends on Hdal activity. K175 was identified as a candidate lysine residue for acetylation by sequence alignment with $S$. cerevisiae Yng2. This was confirmed by the loss of acetylation of Yng2 when we substituted K175 with arginine (K175R), a mutation that blocks acetylation (Figure 4B). The loss of acetylation of $\mathrm{Yng} 2^{\mathrm{K} 175 \mathrm{R}}$ also suggests that K175 is the major acetylated lysine residue of $\mathrm{Yng} 2$ in C. albicans. The K175R mutation led to a reduced level of Yng2, whereas substituting K175 with glutamine (K175Q a mutation mimicking constitutive acetylation) did not cause a detectable change of protein abundance relative to wild type (Figure 4C). Unlike the yng2 deletion mutant, neither $y n g 2^{K 175 R}$ nor $y n g 2^{K 175 Q}$ has any detectable growth defect (unpublished data). We then examined whether Yng2 acetylation affects hyphal development. Both wildtype and $y n g 2^{K 175 R}$ cells developed long hyphae, whereas yng $2^{K 175 Q}$ cells were defective in sustained hyphal development and transcription (Figures 4D,E and S4A), a phenotype similar to that of the hda1 mutant. Therefore, Yng2 deacetylation at K175 is important for sustained hyphal transcription. Conversely, constitutively acetylated Yng2 blocks hyphal filament extension.

If serum-induced $\mathrm{Hdal}$ promoter association leads to Yng2 deacetylation and degradation, the level of promoter-associated Yng2 is expected to decrease during hyphal induction. Indeed, a time course ChIP of Yng2-Myc showed that Yng2 level decreased after $30 \mathrm{~min}$ of hyphal induction (Figures $4 \mathrm{~F}$ and S4B). The level of promoter-associated $\mathrm{Yng} 2^{\mathrm{K} 175 \mathrm{R}}$ showed a similar decrease, whereas the levels of Yng2-Myc in the hda1 mutant and Yng2 ${ }^{\mathrm{K} 175 \mathrm{Q}_{-}}$-Mc stayed unchanged (Figures $4 \mathrm{~F}, 5 \mathrm{C}$, and S4C). The level of Esal, the enzymatic subunit of NuA4, showed a similar decrease at the promoters during hyphal induction (unpublished data).

To determine whether the deacetylation of Yng2 is the major function of Hdal in hyphal development, we generated an hda1 

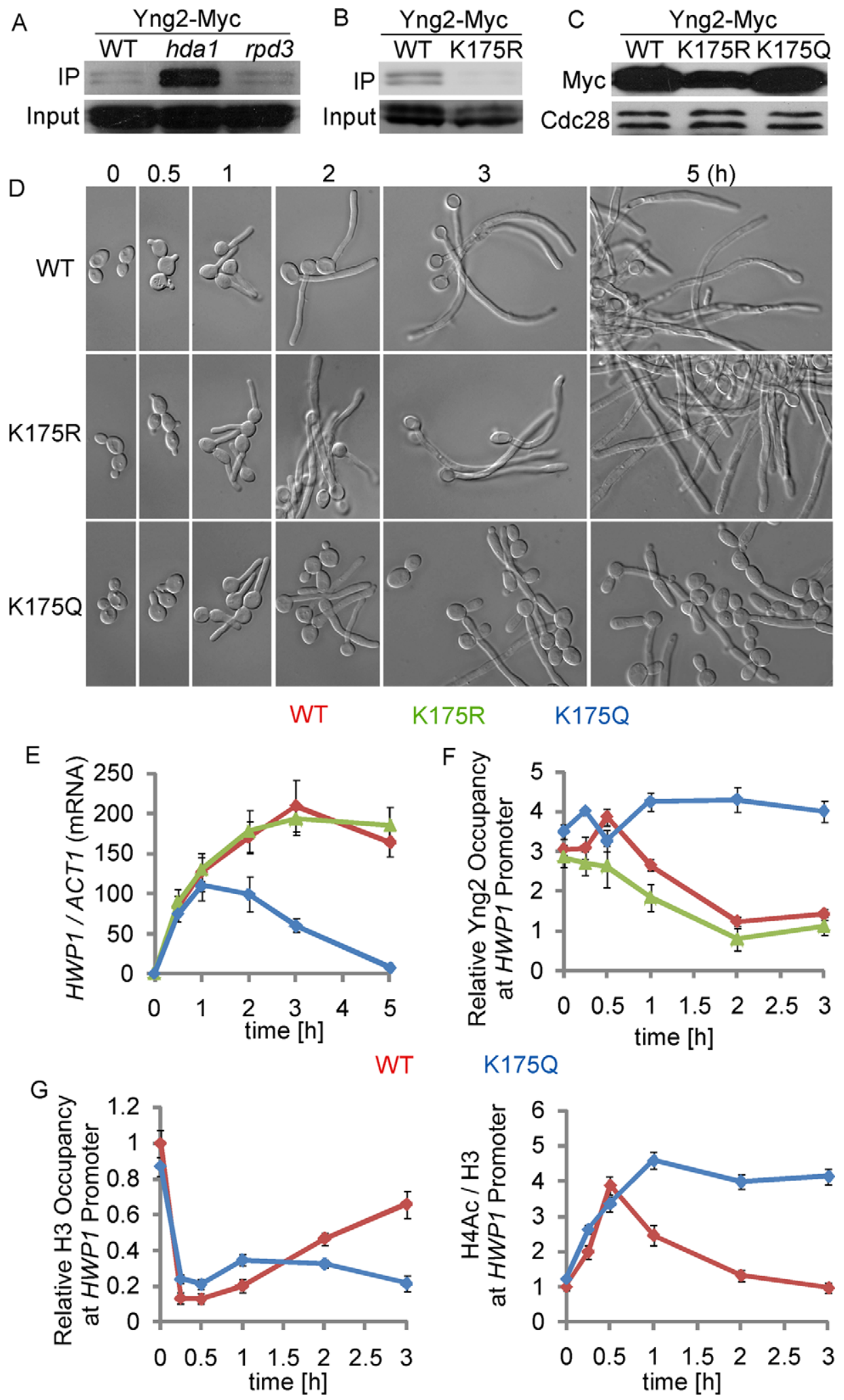

Figure 4. The function of Hda1 in sustained hyphal transcription is mediated through Yng2 deacetylation (A) Yng $2 \mathrm{p}$ is deacetylated through an Hda1-dependent mechanism in vivo. Cells were grown at $30^{\circ} \mathrm{C}$ to $\mathrm{OD}_{600} 0.8$, then WCEs were collected, immunoprecipitated with anti-Ac$\mathrm{K}$, and probed with anti-Myc. (B) K175 is the major acetylated lysine residue of Yng2 in vivo. Substitution of K175 with arginine (K175R) diminishes acetylation of Yng2. (C) Effects of K175 substitutions on Yng2 stability. K175R mutation causes decreased protein abundance of Yng2, while K175Q causes no detectable change. (D, E) Morphology and expression levels of hypha-specific genes in YNG2 (HLY4035), yng $2^{K 175 R}(\mathrm{HLY} 4036)$, and yng $2^{K 1750}$ (HLY4037) cells during hyphal development in YPD with $10 \%$ serum at $37^{\circ} \mathrm{C}$. HWP1, ALS3, and ECE1 mRNA levels were determined by qRT-PCR. The signal obtained from ACT1 mRNA was used as a loading control for normalization. (F) Dynamic dissociation of Myc-tagged Yng2 and yng2 ${ }^{\mathrm{K} 175 \mathrm{R}}$ but not $\mathrm{Yng}^{\mathrm{K}}{ }^{175 \mathrm{Q}}$ from promoters during hyphal development by ChIP with anti-Myc antibodies. (G) ChIP with anti-H3 and anti-acetylated H4 antibodies 
show temporal dynamics in relative levels of $\mathrm{H} 3$ and $\mathrm{H} 4$ acetylation at the promoters of hypha-specific genes during hyphal induction in the $Y N G 2$ (HLY4035) and yng $2^{K 1750}$ mutant (HLY4037) strains. Levels of H3 (relative H3 occupancy; bound/input) are normalized to the respective control DNA at the $A D E 2$ coding sequence region (bound/input). The 0 -h values in the wild-type strain are set to be 1.00 . Acetylation levels normalized with respect to $\mathrm{H} 4$ levels ( $\mathrm{H} 4$ acetylation/H3 occupancy) are calculated by dividing the values for acetylated $\mathrm{H} 4$ with $\mathrm{H} 3$ occupancy values. Data on $A L S 3$ and $E C E 1$ for $(E, F, G)$ are in Figure S4. All data show the average of three independent $\mathrm{qPCR}$ experiments with error bars representing the SEM. doi:10.1371/journal.pbio.1001105.g004

yng2 double mutant and introduced the $y n g 2^{K 175 R}$ mutation into the double mutant. The hda1 yng $2^{K 175 R}$ mutant behaved like $y n g 2^{K 175 R}$ or wild-type cells with no defect in hyphal filament extension and transcription (Figure 5A and 5B). Furthermore, $\mathrm{Hdal}$ is required for the reduction of promoter-associated Yng2, and the $y n g 2^{K 175 R}$ mutant completely bypassed this requirement of Hdal (Figures 5C and S5). Therefore, the function of Hdal in sustained hyphal transcription is mediated completely through the deacetylation of Yng2 at K175. In addition, the level of promoterassociated $\mathrm{Yng} 2^{\mathrm{K} 175 \mathrm{R}}$ decreased in the hda1 mutant as did $\mathrm{Yng} 2 \mathrm{in}$ wild-type cells during hyphal elongation (Figures 5C and S5), suggesting that Hdal deacetylation of $\mathrm{Yng} 2$ at the promoters led to the eviction of Yng2 and Esal from the promoters of hyphaspecific genes.

To understand the effect of Yng2 K175 deacetylation on promoter chromatin, we determined temporal changes in histone levels and $\mathrm{H} 4$ acetylation at the UAS regions of hypha-specific promoters during hyphal induction (Figures $4 \mathrm{G}$ and $\mathrm{S} 4 \mathrm{D}$ ). Changes in $\mathrm{H} 3$ levels indicated rapid nucleosome disassembly during hyphal initiation and nucleosome reassembly during hyphal maintenance. The observed decrease in H3 occupancy and transcriptional induction within $30 \mathrm{~min}$ of hyphal induction is consistent with the notion that transcriptional activation correlates with reduced nucleosome occupancy [57]. In contrast, nucleosome reassembly during hyphal elongation seemed important for sustained hyphal transcription, as yng $2^{K 175 Q}$ cells showed a specific defect in nucleosome reassembly without any detectable impairment in initial nucleosome disassembly. Our data suggest that nucleosome reassembly during hyphal maintenance may function in preventing $\mathrm{Nrg} 1$ from binding to the promoters.

\section{Reduced TOR Signalling Led to Hda1 Promoter Recruitment and Sustained Hyphal Development}

$y n g 2^{K 175 Q}$ cells exhibited normal hyphal initiation in several hyphal growth media in addition to serum (Table S1B) and were defective in sustained hyphal development in all media that can support prolonged hyphal development (Figure 6A). This suggested that the Hdal-mediated deacetylation of Yng2 was
A

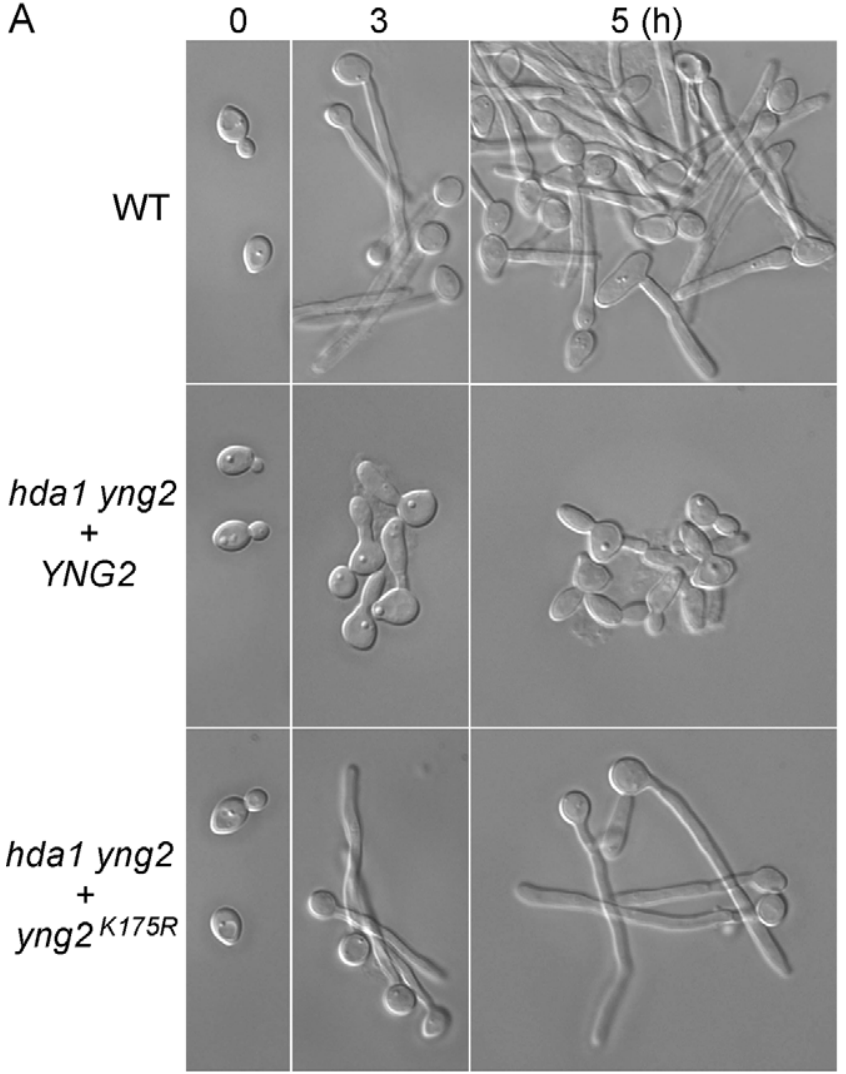

B
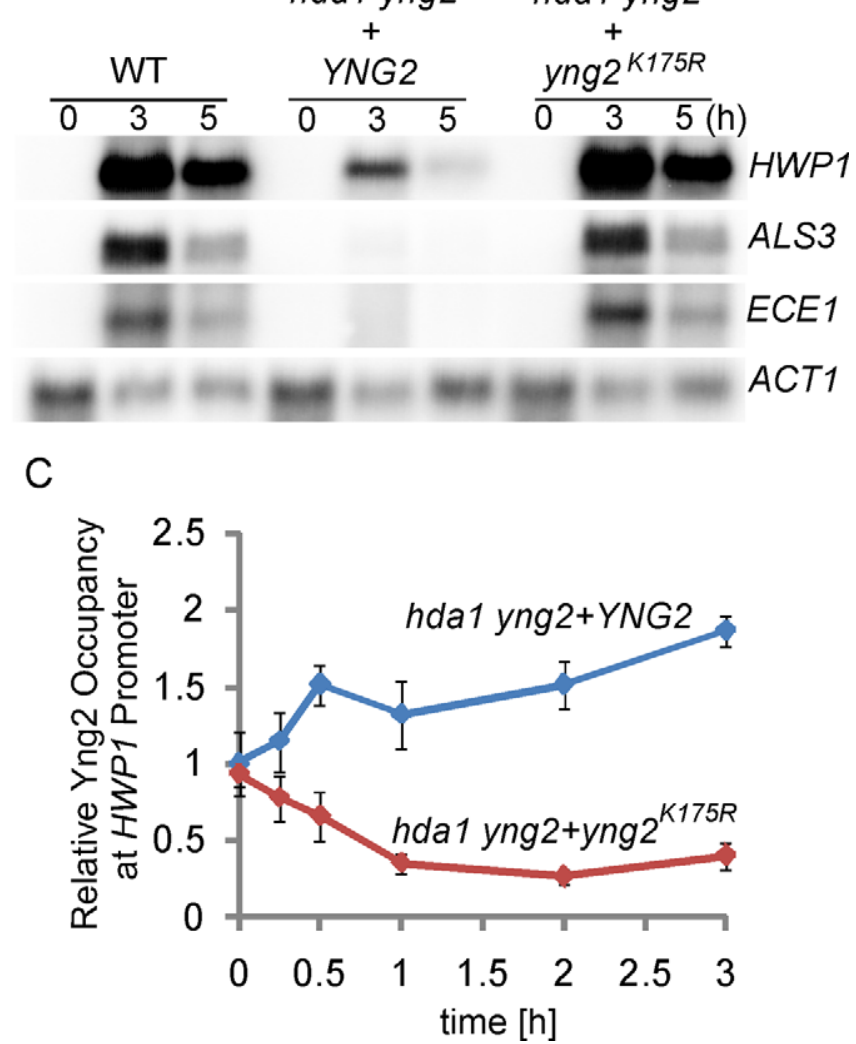

Figure 5. $y n g 2^{K 175 R}$ mutation suppresses the deficiency of $h d a 1 / h d a 1$ in sustained expression of hypha-specific genes. Morphology (A) and Northern analysis (B) of wild type and hda1/hda1 yng2/yng2 transformed with YNG2 or yng $2^{K 175 R}$ during hyphal induction as in Figure 3. Northern analysis was carried out using $12.5 \mu \mathrm{g}$ RNA and probes to the indicated filament-specific transcripts. The ACT1 transcript is included as a loading control. (C). Kinetics of Yng2-Myc and Yng2-Myc-K175R promoter binding in hda1/hda1 mutants by ChIP with anti-Myc. Data on ALS3 and ECE1 for (C) are in Figure S5. The ChIP data show the average of three independent QPCR experiments with error bars representing the SEM.

doi:10.1371/journal.pbio.1001105.g005 
A

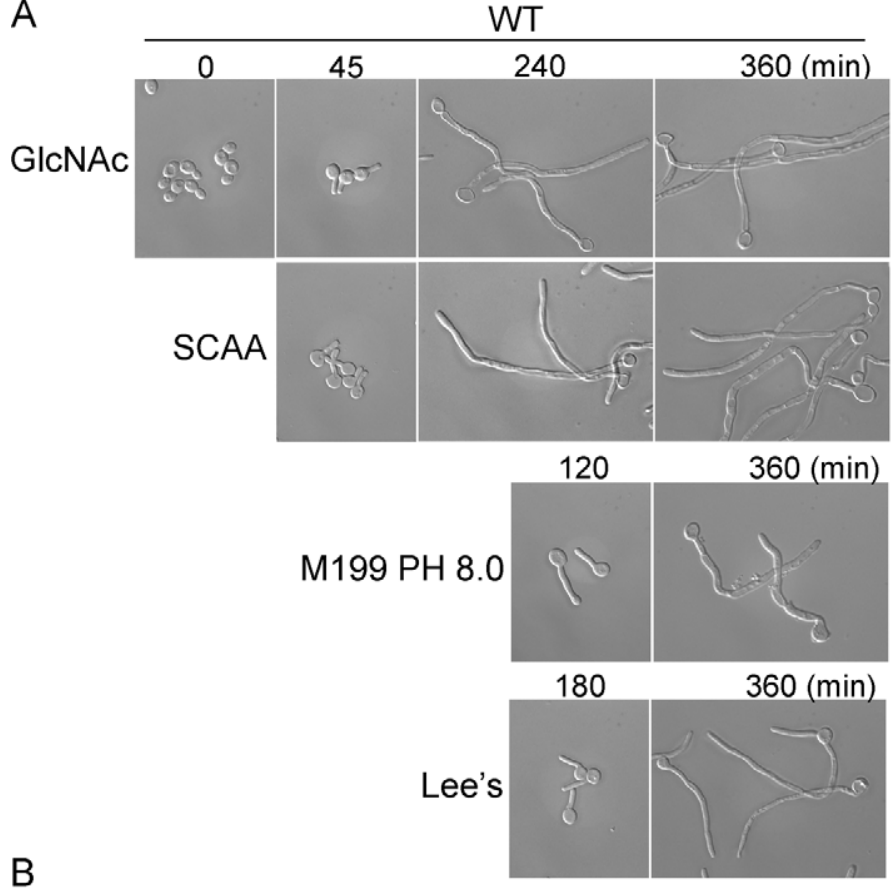

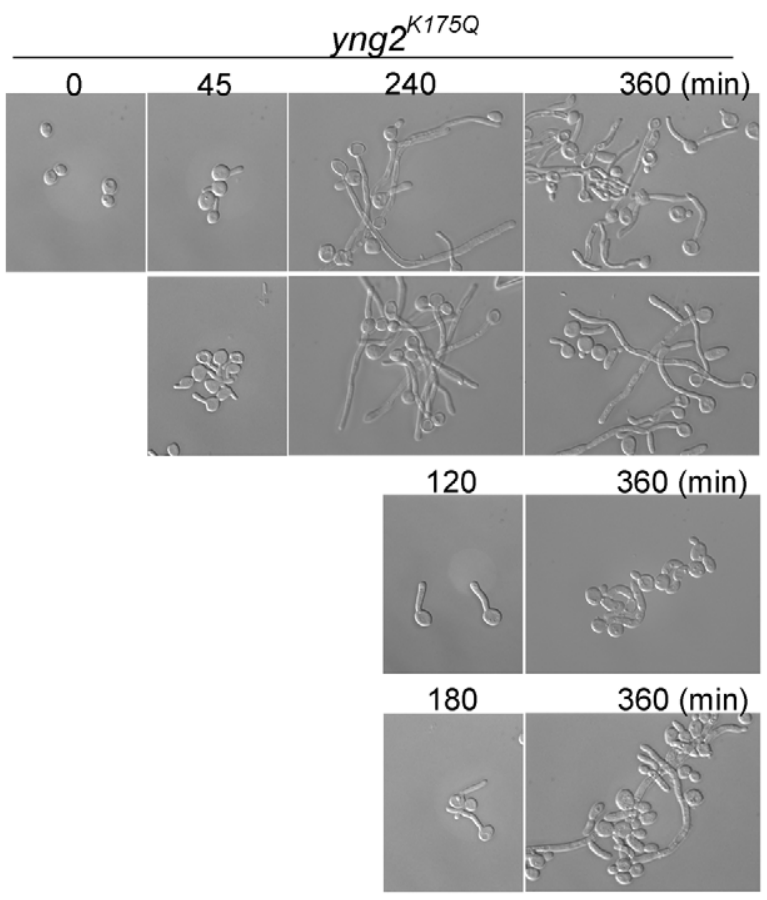

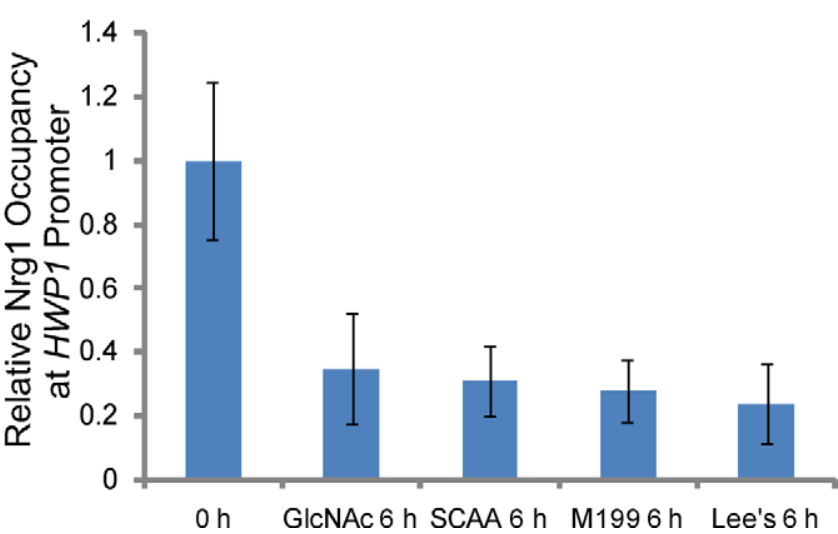

Figure 6. $y n g 2^{K 1750}$ mutant is defective in hyphal elongation in nutrient-poor media. (A) Cell morphology of wild-type (HLY4035) and yng ${ }^{K 1750}$ (HLY4037) cells grown at $37^{\circ} \mathrm{C}$ in YEP+GIcNAc, SCAA, M199 pH 8.0, or Lee's with mannitol for indicated times. (B) ChIP with anti-Myc antibodies in wild-type cells carrying Hda1-Myc (HLY4033) or Nrg1-Myc (HLY3922). qPCR was used to quantitate ChIP DNA with primers at the UAS region of HWP1 under the indicated conditions. The ChIP data show the average of three independent qPCR experiments with error bars representing the SEM.

doi:10.1371/journal.pbio.1001105.g006

required for hyphal maintenance in those media. In addition, Hdal was found to be associated with the promoters of hyphaspecific genes, whereas $\mathrm{Nrg} 1$ promoter-association was inhibited after $6 \mathrm{~h}$ of growth in those conditions (Figure 6B). Interestingly, those media tend to be nutrient-poor. One commonly used synthetic nutrient-poor medium for hyphal growth is Lee's medium [58]. It contains glucose, ammonium sulfate, and many amino acids, but lacks glutamine, a preferred nitrogen source. The rapamycin-sensitive Torl kinase is a central regulator of cell growth in response to nitrogen and amino acid availability in yeast [59] and is conserved in C. albicans [41]. We hypothesized that a nutrient-poor medium, such as Lee's, is sensed by $C$. albicans cells as nitrogen limiting, and therefore leads to reduced Torl activity. If so, treatment of C. abicans cells with rapamycin in a rich medium should mimic a nutrient-poor medium for sustained hyphal development. As predicted, treatment of C. albicans cells with a sublethal concentration of rapamycin after hyphal initiation at $37^{\circ} \mathrm{C}$ led to sustained hyphal filamentation in rich medium
(Figure 7A). A higher concentration of rapamycin, however, slowed cell growth and hyphal elongation (unpublished data), consistent with the finding that rapamycin inhibits filamentation on nutrient-poor media [41]. Sustained hyphal filamentation was also observed at $30^{\circ} \mathrm{C}$ with sublethal levels of rapamycin after cells were first grown at $37^{\circ} \mathrm{C}$ for hyphal initiation (Figure 7A). The effect of rapamycin on hyphal maintenance was only observed after hyphal initiation. Cells treated with rapamycin at $30^{\circ} \mathrm{C}$ did not form hyphae (Figure 7B). We further showed that the effect of Torl on hyphal maintenance was mediated through the promoterrecruitment of $\mathrm{Hdal}$, as $y n g 2^{K 175 Q}$ cells were defective in sustained hyphal filamentation in rapamycin (Figure 7C). In addition, Hdal was recruited to the promoters of hypha-specific genes in cells treated with rapamycin in rich medium, whereas $\mathrm{Nrgl}$ was excluded from the promoters (Figure 7D). The phenotypes of the yng $2^{K 175 Q}$ cells and patterns of promoter association for Hdal and $\mathrm{Nrg} 1$ are similar between poor medium and rapamycin treatment (Figures 6 and 7). Furthermore, a mutant with a hyperactive TOR 


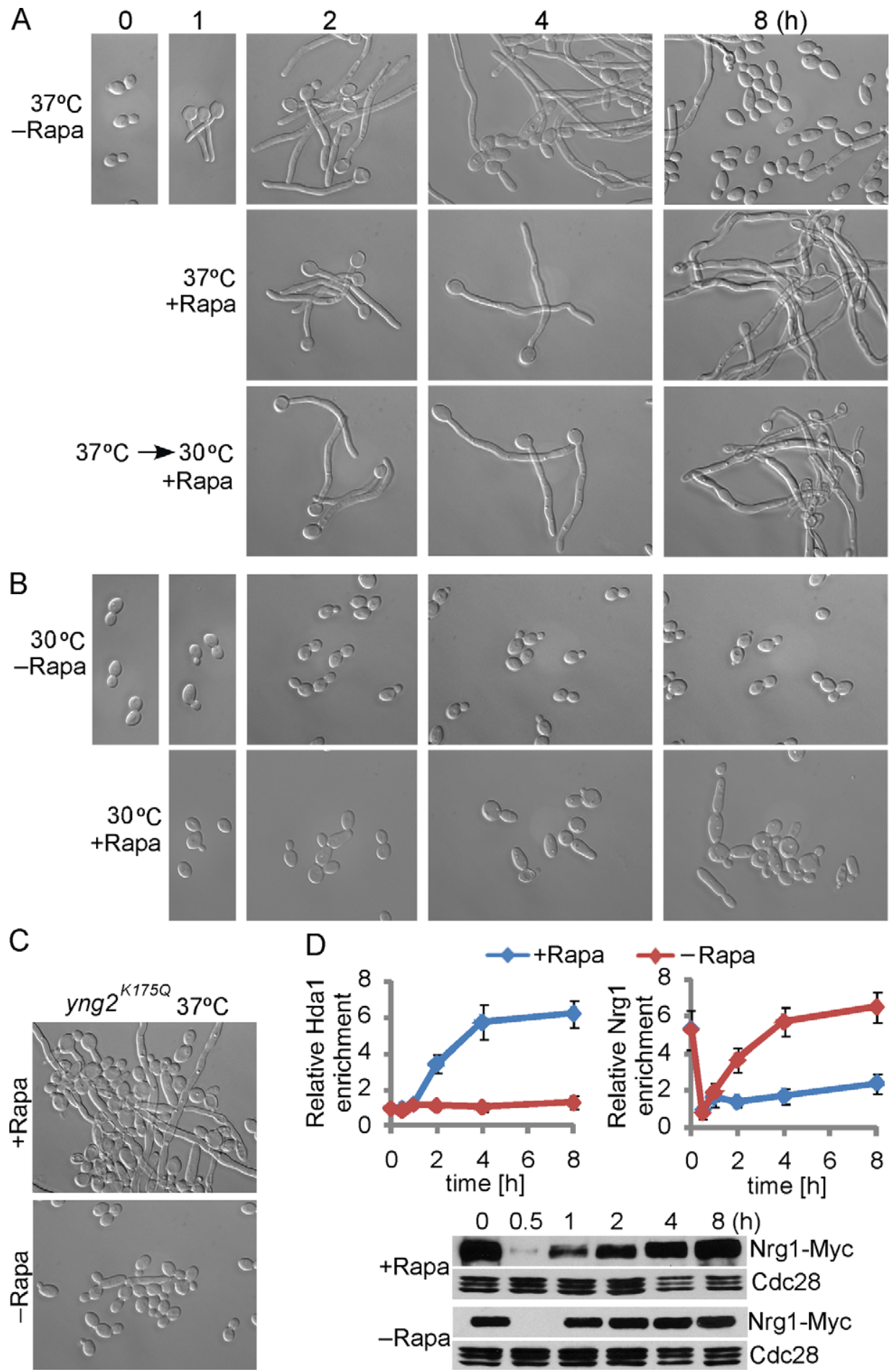

Figure 7. Reduced Tor1 signaling allows sustained hyphal elongation through promoter recruitment of Hda1. (A) Treatment of C. albicans cells with rapamycin after hyphal initiation at $37^{\circ} \mathrm{C}$ led to sustained hyphal filamentation in rich medium. Wild-type (HLY4035) cells were grown in YPD medium at $30^{\circ} \mathrm{C}$ overnight and were diluted at $1: 250$ into pre-warmed YPD medium at $37^{\circ} \mathrm{C}$ or grown at $37^{\circ} \mathrm{C}$ for $1 \mathrm{~h}$ and then transferred to $30^{\circ} \mathrm{C} .5 \mathrm{nM}$ rapamycin was added after $1 \mathrm{~h}$ to half of the samples. Cells were collected at $0 \mathrm{~h}, 1 \mathrm{~h}, 2 \mathrm{~h}, 4 \mathrm{~h}$, and $8 \mathrm{~h}$ for cell morphology. (B) Wild-type cells grown in YPD at $30^{\circ} \mathrm{C}$ with and without $5 \mathrm{nM}$ rapamycin for the indicated hours. (C) yng $2^{K 1750}$ (HLY4037) cells were grown in YPD at $37^{\circ} \mathrm{C}$ for 8 h. $5 \mathrm{nM}$ rapamycin was added after $1 \mathrm{~h}$ to half of the samples. (D) Nrg1 protein levels and Nrg1-myc or Hda1-myc promoter association during hyphal growth in YPD at $37^{\circ} \mathrm{C}$ with and without $10 \mathrm{nM}$ rapamycin. ChIP DNA were quantitated by qPCR with primers at the UAS region of HWP1. The ChIP data show the average of three independent qPCR experiments with error bars representing the SEM. doi:10.1371/journal.pbio.1001105.g007

pathway in C. albicans has recently been shown to be unable to form hyphae in a nutrient-poor medium, and rapamycin can rescue the filamentation defect [60]. Therefore, maintenance of hyphal development requires reduced Torl signaling in response to nutrient-poor media and growth conditions. The reduced Tor 1 signaling leads to the recruitment of Hdal to the promoters of 
hypha-specific genes for promoter remodeling that prevents $\mathrm{Nrg} 1$ from binding.

\section{Temporal Connection between Hyphal Initiation and Maintenance}

To uncover potential molecular connections between the initiation phase and elongation phase of hyphal development, we asked whether serum could induce the promoter recruitment of Hdal without the initiation phase. We found that Hdal did not bind the promoters of hypha-specific genes when cells were grown at $30^{\circ} \mathrm{C}$ in the presence of serum (Figure $8 \mathrm{~A}$ ). Therefore, serum is not sufficient to induce the promoter recruitment of $\mathrm{Hdal}$ without the initiation step. We further showed that raising the temperature to $37^{\circ} \mathrm{C}$ during inoculation was a prerequisite for subsequent recruitment of Hdal to the promoters (Figure 8A). Since Nrgl was temporally cleared when cells were inoculated into fresh medium at $37^{\circ} \mathrm{C}$, a requirement for hyphal initiation, we then examined whether serum could induce promoter recruitment of Hdal independent of temperature in the $n r g 1$ deletion mutant by a time course ChIP experiment. Hdal was found to bind the HWP1 promoter in the presence of serum in $n r g 1$ mutants in either $25^{\circ} \mathrm{C}$ or $37^{\circ} \mathrm{C}$ (Figure $8 \mathrm{~B}$ ). This result suggests that $\mathrm{Nrgl}$ removal is required and sufficient for serum-induced Hdal promoter recruitment for the sustained hyphal transcriptional program. This also predicts that the sustained hyphal transcriptional program could only be established within the time window of reduced $\mathrm{Nrgl}$ when cells had just been inoculated into fresh media at $37^{\circ} \mathrm{C}$. Indeed, addition of serum after $2 \mathrm{~h}$ of hyphal induction at $37^{\circ} \mathrm{C}$ in YPD could not sustain hyphal development (Figure 8C). Similar to serum, rapamycin could sustain hyphal growth only when added during hyphal initiation. Adding rapamycin after $2 \mathrm{~h}$ of hyphal induction showed no effect on hyphal maintenance
A

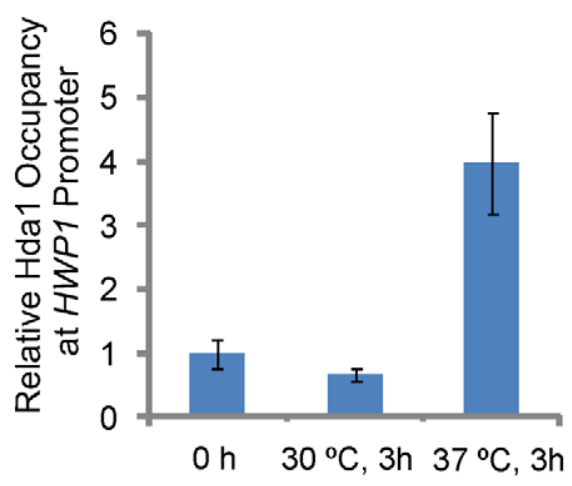

B

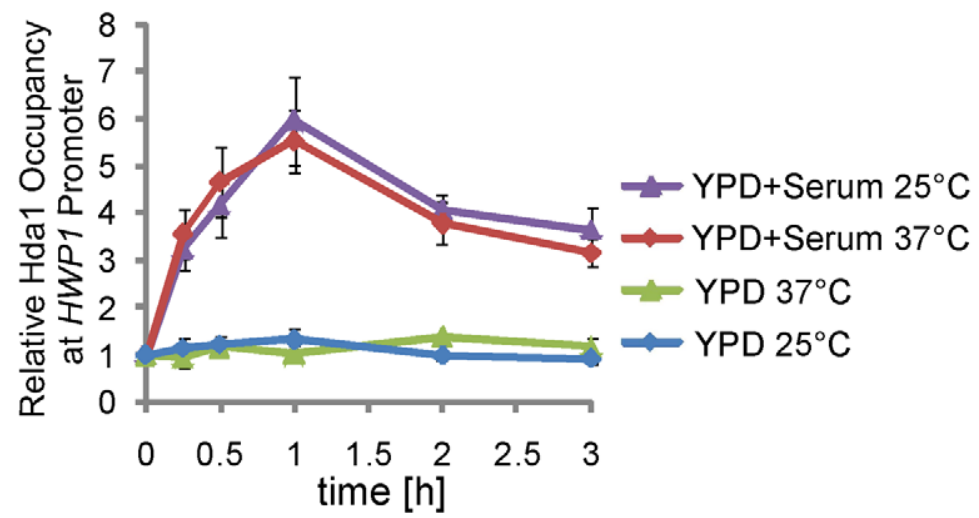

C

Time that serum or rapamycin was added after inoculation

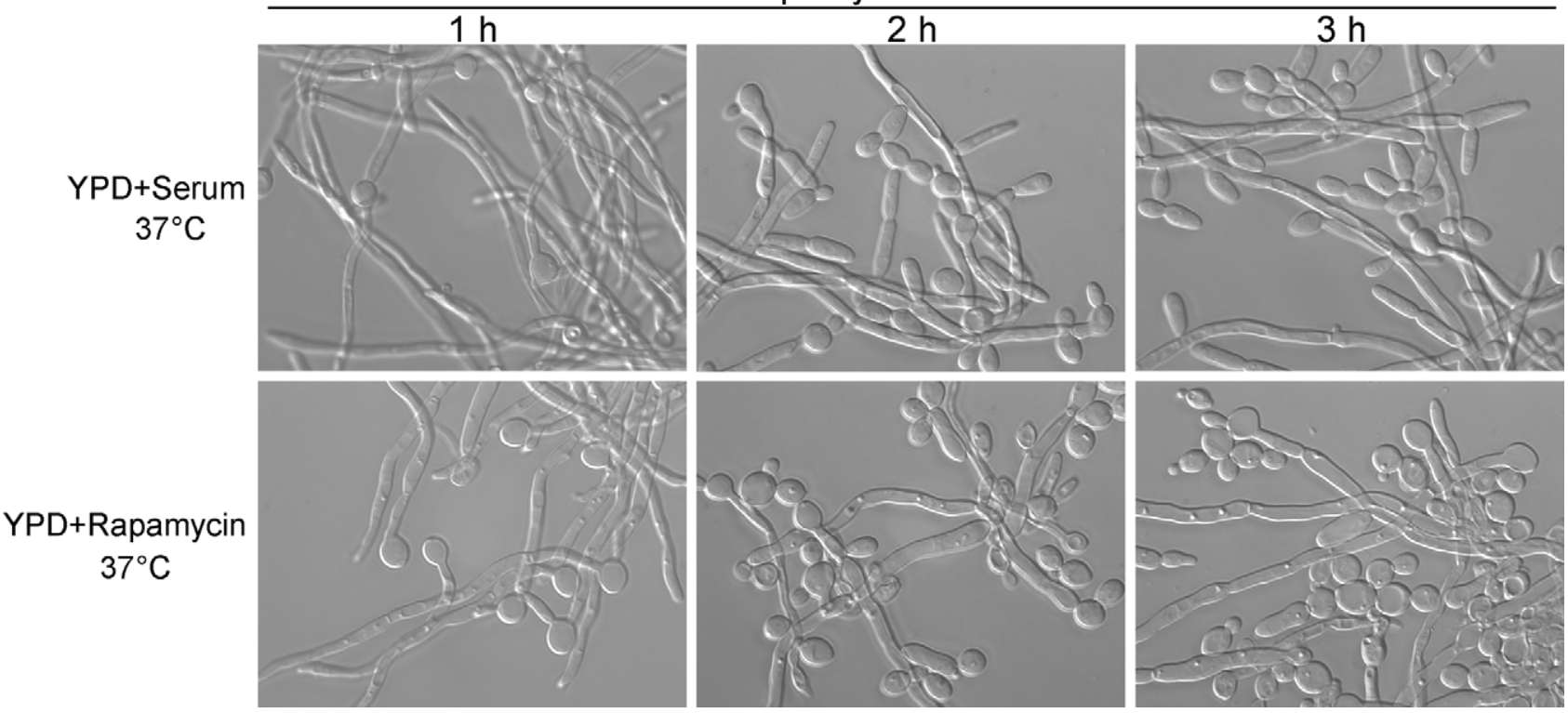

Figure 8. The sustained hyphal growth program could only be established during the time window of reduced Nrg1. (A) Serum is not sufficient to induce the promoter recruitment of $\mathrm{Hda} 1$ without hyphal initiation at $37^{\circ} \mathrm{C}$. ChIP with anti-Myc antibodies in wild-type cells carrying Hda1-Myc in YPD with 10\% serum under indicated conditions. Primers at the UAS region of HWP1 were used. (B) Nrg1 removal is required and sufficient for serum-induced Hda1 promoter recruitment. The ChIP time course was carried out with anti-Myc antibodies in $n r g 1 / n r g 1$ cells carrying Hda1-Myc under the indicated conditions. Cells were collected at $0 \mathrm{~min}, 15 \mathrm{~min}, 30 \mathrm{~min}, 1 \mathrm{~h}, 2 \mathrm{~h}$, and $3 \mathrm{~h}$. The ChIP data show the average of three independent qPCR experiments with error bars representing the SEM. (C) Serum and rapamycin are unable to sustain hyphal development when they are added to YPD medium $2 \mathrm{~h}$ after inoculation at $37^{\circ} \mathrm{C}$. Cells from overnight cultures in YPD at $30^{\circ} \mathrm{C}$ were diluted at 1:250 into pre-warmed YPD medium at $37^{\circ} \mathrm{C} ; 10 \%$ serum or $5 \mathrm{nM}$ rapamycin were added at $1 \mathrm{~h}, 2 \mathrm{~h}$, or $3 \mathrm{~h}$ after inoculation, respectively. The total incubation time is $5 \mathrm{~h}$ for serum and $8 \mathrm{~h}$ for rapamycin experiments.

doi:10.1371/journal.pbio.1001105.g008 
(Figure 8C). Together, our data show a clear temporal connection between hyphal initiation and maintenance: Hdal can be recruited to the promoters of the hypha-specific genes only when Nrgl level is low. The time period of reduced Nrgl during hyphal initiation can be viewed as a window of opportunity for establishing the hyphal transcription program. The strength and duration of the cAMP signal in each cell is expected to affect the commitment to the hyphal transcriptional program; once committed, the cAMP-PKA pathway activation is no longer needed. This is consistent with the observation that farnesol only inhibits germ-tube formation and has no effect on hyphal elongation [39]. The duration of hyphal development is determined by nutrient conditions through the TOR pathway.

\section{Discussion}

This study shows that the yeast to hyphae morphogenetic switch consists of two temporally related phases of regulation of the promoter chromatin of hypha-specific genes: the first for initiation and the second for maintenance of hyphal development (Figure 9). Initiation requires the cAMP-PKA pathway and maintenance requires reduced Tor 1 signaling. A spike of cAMP-PKA activation [10] and release from the inhibition of quorum sensing molecules, such as farnesol, initiate hyphal development by transiently clearing Nrgl protein. The duration of hyphal development is under the regulation of Torl by controlling promoter access to Nrgl through promoter recruitment of Hdal. Therefore, hyphal maintenance requires active sensing of growth environments. Hyphal cells convert to yeast when nutrients are replete. This provides an underlying mechanism for the plasticity of dimorphism. Importantly, the sustained transcriptional program, as measured by promoter recruitment of Hdal, can only be established during the time period when Nrgl is absent. This provides a temporal link at the molecular level between the two phases of hyphal development. This is the first example of a temporal integration of two major nutrient-sensing cell growth pathways in development. Temporal restriction in development of multicellular organisms is common. For example, nutritional control of the reproductive status in honeybees is restricted by a critical "decision-making" period in larval development [61]. Our study also provides a mechanism for how a transient signal, such as a cAMP spike, can make a long-lasting impact in transcriptional reprogramming during development. Such control of cellular development by a burst of proliferating signal, followed with sustained action of reduced TOR signaling has been observed for memory T-cell differentiation [62]. The TOR level is also critical for maintaining stem cell homeostasis [63-65]. Temporal regulation of cell fate by different signaling pathways is likely

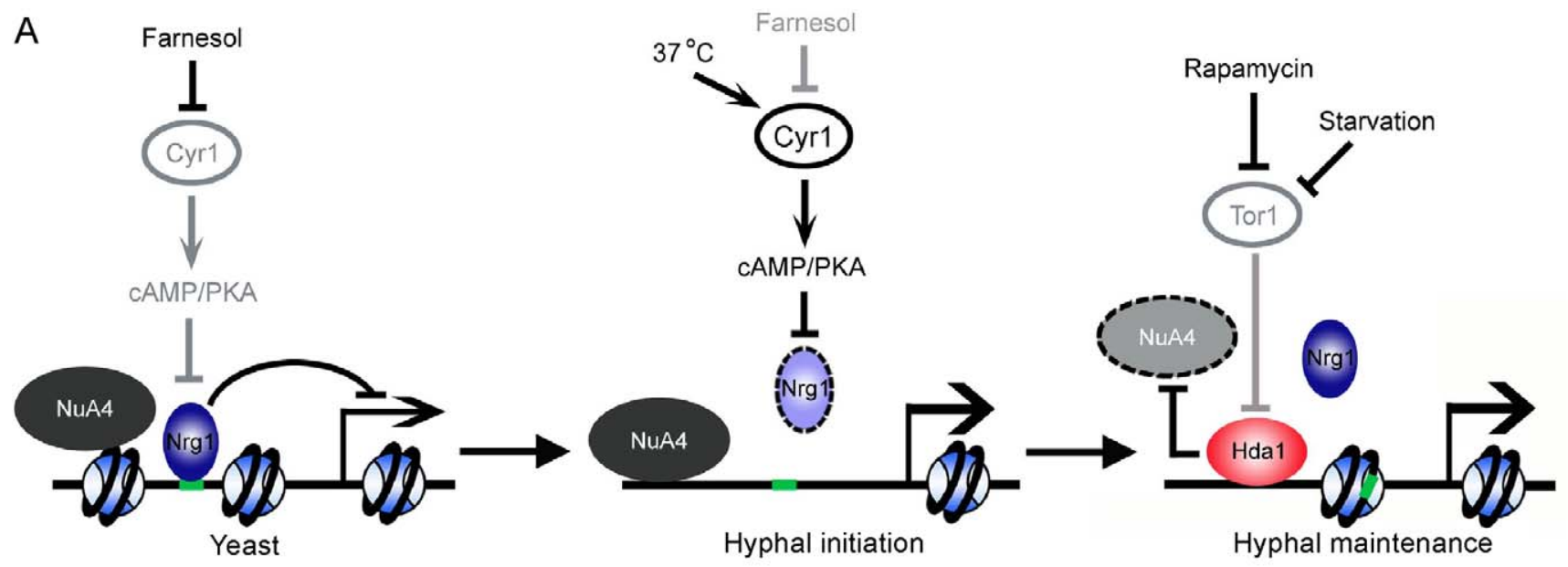

B

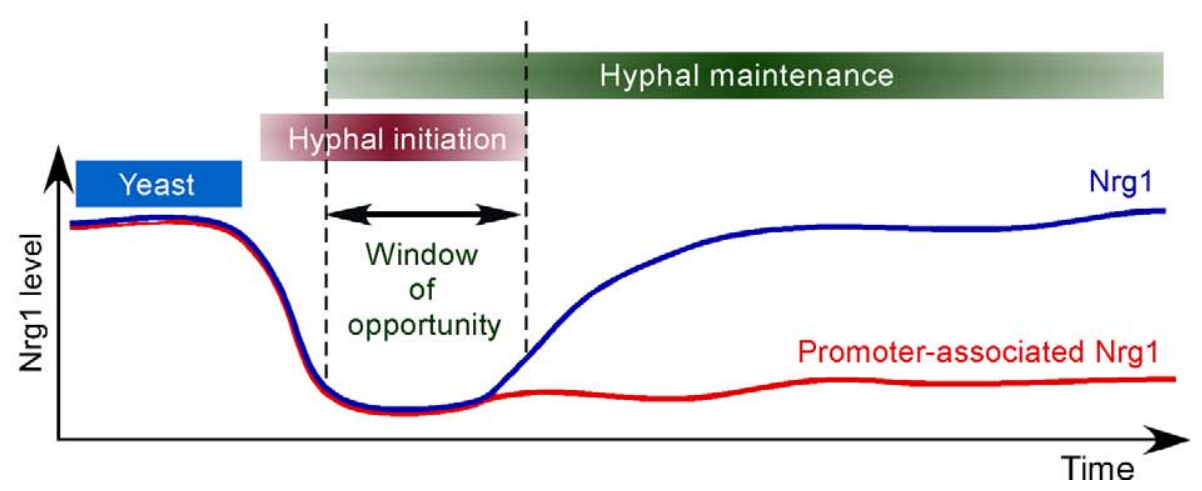

Figure 9. A schematic diagram depicting initiation and maintenance of hyphal development. (A) Hyphal development in C. albicans requires two phases of sequential changes in the promoter chromatin of hypha-specific genes. Initiation requires a rapid but temporary disappearance of $\mathrm{Nrg} 1$ in response to the activation of the CAMP-PKA pathway, while the duration of hyphal development is under the regulation of the TOR pathway by controlling promoter access to Nrg1 through recruitment of Hda1. Black lines represent active regulatory relationships; grey lines represent relationships that are inactive. Dashed circles represent degraded proteins. (B) Hda1 is recruited only during the time period when Nrg1 is absent. This provides a mechanism for temporal integration of two major nutrient-sensing cell growth pathways in development. doi:10.1371/journal.pbio.1001105.g009 
common in development of multicellular organisms. Coupling two signaling pathways through the regulation of promoter chromatin provides a unique mechanism.

Initiation of the hyphal transcriptional program requires temporary removal of $\mathrm{Nrgl}$ protein. The timing, duration, and extent of the $\mathrm{Nrgl}$ down-regulation are well correlated with timing and efficiency of hyphal initiation and are sensitive to multiple factors, including the state of inoculating cells (e.g., stationary cells), fold of dilution at inoculation, media, and temperature. Of those, a rise in temperature to $37^{\circ} \mathrm{C}$ and release from farnesol inhibition are essential for the clearance of $\mathrm{Nrgl}$ and hyphal initiation. The cAMP-PKA pathway is required for the downregulation of $\mathrm{Nrg} 1$, consistent with the concept that Cyrl is a hub that integrates many of the hyphal inducing signals [31-33,40]. Efg 1 and Flo8 are both required for the down-regulation of $\mathrm{Nrg} 1$, but a $\mathrm{T} \rightarrow \mathrm{A}$ or $\mathrm{T} \rightarrow \mathrm{E}$ mutation at the sole conserved PKA site in Efgl (T206) does not reduce or enhance hyphal gene expression (our unpublished data). If Efgl activity is directly regulated by PKA phosphorylation, the regulation is not essential or sufficient for the induction of hypha-specific genes. Efgl and Flo8 also function directly on the promoters of hypha-specific genes [16,50], and the tup 1 mutant cannot bypass the requirement of Efg 1 for hyphal transcription [66]. Our finding that $\mathrm{Nrgl}$ is negatively regulated by the cAMP-PKA pathway and by farnesol is intriguing. The $n r g 1$ mutant produces 19-fold more farnesol than the parent wild-type strain [67], and farnesol in turn impacts activity of the Cyr 1 pathway [40]. Together, these findings provide a loop of three negative feedback regulations from farnesol to Cyrl, to Nrgl, and then back to farnesol. Removal of farnesol during inoculation will lead to activation of the cAMP-PKA pathway and clearance of $\mathrm{Nrg} 1$ (Figure 9). Lack of $\mathrm{Nrg} 1$ in turn is expected to lead to increased production of farnesol, and a new balance among the components in the feedback loop will be achieved. Another intriguing finding is that the sustained hyphal transcriptional program can only be established during the time window of low $\mathrm{Nrg} 1$. Commitment to the hyphal program is therefore determined by the strength and duration of $\mathrm{Nrgl}$ downregulation in each cell. If the duration is not long enough to establish the Hdal-mediated chromatin remodeling, the cell will be in the yeast growth phase. This explains the cell-to-cell variation and experiment-to-experiment variation in hyphal induction in a given culture, and why yeast and hyphal cells can exist in the same culture. Our model explains a widely accepted observation that the quality of the initial hyphal induction is important for the fate of hyphal development. It also provides a molecular mechanism for why farnesol only inhibits germ-tube formation and has no effect on hyphal elongation [39].

Our finding of temporally coupled chromatin remodeling under reduced Tor 1 signaling for sustained hyphal transcription provides a molecular mechanism for why nutrient-poor media are typical media for sustained hyphal development. It has been puzzling how C. albicans can respond to both rich and poor media and undergo hyphal development. Rich medium with serum is the most robust medium for hyphal induction, and this is consistent with the requirement of the cAMP-PKA pathway for hyphal growth. Hyphal induction in nutrient-poor media also requires an activation of the cAMP-PKA pathway, mostly through the release from farnesol inhibition. But sustained hyphal growth requires a nutrient limitation. Considering the conserved functions of Torl in nutrient sensing and growth regulation from yeast to human, involvement of Torl in hyphal development has been studied and implicated $[41,60,68]$. However, rapamycin cannot induce hyphal development, making it hard to reconcile if the cAMP-PKA and Torl pathways function in parallel. We find that rapamycin can lead to the promoter recruitment of Hdal only during hyphal initiation in the absence of $\mathrm{Nrgl}$. The temporal coupling of the two pathways through the regulation of the promoter chromatin of hypha-specific genes explains why rapamycin by itself cannot induce hyphae. Since serum can sustain hyphal development in YPD, we suspect it may contain certain components that are inhibitory to the Torl pathway. The nuclear localization and the activity of several nutrient-responsive transcription factors are regulated by Torl-mediated phosphorylation in yeast [69]. It is possible that a Tor1-regulated transcription factor is necessary for the recruitment of Hdal to the promoters of hypha-specific genes. Further studies are needed to address this. In addition to Hdalmediated chromatin remodeling for sustained hyphal transcription, there is a built-in positive feedback on the hyphal transcriptional program that is also important for sustained hyphal development. The transcription factor Ume6, specifically expressed during hyphal development, controls the level and duration of hypha-specific genes and is important for hyphal elongation [53,70,71]. The strength of the cAMP and Torl signaling determines the level and duration of UME6 expression, which in turn dictates the extent of hyphal morphogenesis. Hyphal morphogenesis and cell chain formation is under the control of another hypha-specific gene that encodes the $G_{1}$ cyclin-related protein Hgcl [72-76].

Our study should provide insights into understanding C. albicans pathogenesis. Hyphal initiation is tightly linked to release from quorum-sensing molecules, a condition parallel to dispersion of $C$. albicans cells from biofilms in human hosts, which is a major cause of disseminated candidiasis [77]. Invasive candidiasis is the most common invasive fungal infection among organ transplant recipients [78]. Rapamycin is given to transplant patients, especially renal transplant patients, to prevent organ rejection. One speculation from our finding is that rapamycin, known for its antifungal activity, may also facilitate hyphal development and invasive infections. The existence of extensive hyphae in various deep-seeded infection sites also suggests that those host environments may be perceived as stressful or starvation conditions by Candida. Our findings provide molecular mechanisms for how $C$. albicans adapts to varied host environments and develops from a benign commensal into a disseminated invasive disease.

We have previously noticed the dynamic increase and decrease in H4 acetylation concomitant with nucleosome disassembly and reassembly at the promoters of hypha-specific genes during hyphal induction [50]. The significance of the temporal dynamic regulation in promoter chromatin is not clear. Here we find the sequential dissociation of $\mathrm{Rpd} 3$ and association of $\mathrm{Hdal}$ to the promoters is concomitant with the dynamic changes in $\mathrm{H} 4$ acetylation. Importantly, we show that promoter-associated Hdal decreases $\mathrm{H} 4$ acetylation level by deacetylating Yng2 and evicting Yng2 and Esal of the NuA4 HAT module from the promoters, leading to nucleosome reassembly, inhibition of $\mathrm{Nrgl}$ binding, and sustained hyphal transcription. This provides an example for Hdal in gene activation and $\mathrm{NuA} 4$ in gene repression. The function of NuA4 in repression here is revealed by the use of $y n g 2^{K 175 Q}$ and $y n g 2^{K 175 R}$ mutations. Temporal analysis of promoter chromatin also delineates different functions of HDACs and NuA4 in transcription. It shows that nucleosome reassembly does not necessarily correlate with transcriptional repression; it can also be used for gene activation by restricting access of repressors. In $S$. cerevisiae, Yng2 is deacetylated by $\mathrm{Rpd} 3$ and a similar temporal dynamic $\mathrm{H} 4$ acetylation is observed at DNA double-stranded breaks concomitant with the sequential recruitment of NuA4 and Rpd3 [56]. Such temporal dynamic regulation of chromatin is likely also used in transcription in $S$. cerevisiae. Indeed $\mathrm{Rpd} 3$ is required for the expression of several 
environment-responsive genes [79-82]. Active functions of HDACs in gene expression are not limited to yeast. A recent genome-wide mapping of HAT and HDAC binding sites in human T cells shows that all HDACs analyzed are associated with active genes and positively correlated with transcription, with some HDACs mainly in the promoter regions and other HDACs in both the promoter and gene body regions [83]. Inhibition of HDAC activity with HDAC inhibitor treatment causes increases in acetylation in the active genes, suggesting that the majority of HDAGs function to reset chromatin by removing acetylation at active genes [83]. The temporal dynamic NuA4 regulation by HDACs shown in this study provides an attractive mechanism likely used for temporal integration of different signals in transcriptional reprogramming during development.

\section{Materials and Methods}

\section{Media and Growth Conditions}

C. albicans strains were routinely grown at $30^{\circ} \mathrm{C}$ in YPD $(2 \%$ Bacto peptone, $2 \%$ dextrose, $1 \%$ yeast extract). Transformants were selected on synthetic medium ( $2 \%$ dextrose, $0.17 \%$ Difco yeast nitrogen base w/o ammonium sulfate, $0.5 \%$ ammonium sulfate and auxotrophic supplements). M199 medium (SigmaAldrich) was buffered at pH 8 using 150 mM HEPES. Hyphal induction was performed as follows. Strains were grown overnight in liquid YPD at $30^{\circ} \mathrm{C}$, pelleted, washed twice in PBS, resuspended in an equal volume of PBS, and diluted 1:100, unless otherwise indicated, into YPD+10\% serum (Sigma-Aldrich), Lee's medium

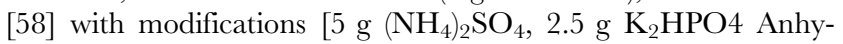
drous, $5 \mathrm{~g} \mathrm{NaCl}, 0.5 \mathrm{~g}$ alanine, $1.3 \mathrm{~g}$ leucine, $1 \mathrm{~g}$ lysine, $0.1 \mathrm{~g}$ methionine, $0.072 \mathrm{~g}$ ornithine, $0.5 \mathrm{~g}$ proline, $0.5 \mathrm{~g}$ threonine, $10 \mathrm{ml} 20 \mathrm{mg} / \mathrm{ml} \mathrm{MgSO}_{4}, 1 \mathrm{ml} 2 \mathrm{mg} / \mathrm{ml}$ biotin, with $\mathrm{pH}$ adjusted to 7.0 , with either $1 \%$ glucose or mannitol, filled to $1 \mathrm{~L}$ with water], YEP+2\% N-acetyl-glucosamine (Sigma-Aldrich), SCAA medium [15] with modifications [2\% Casein Hydrolysate broth (Sigma-Aldrich), $0.17 \%$ Difco yeast nitrogen base w/o ammonium sulfate], Spider medium [1\% mannitol, $1 \%$ nutrient broth, $0.2 \%$ $\mathrm{K}_{2} \mathrm{HPO}_{4}$, pH 7.2 before autoclaving] [84], M199 pH 8 or $\mathrm{YPD}+5 \mathrm{nM}$ rapamycin. Cultures were grown at $37^{\circ} \mathrm{C}$.

\section{Plasmid and Strain Construction}

The C. albicans strains used in this study are listed in Dataset S1. Primer sequences are listed in Dataset S2. HDho15 and RPho19 [54] were streaked on 5-fluoro-orotic acid-containing medium to generate HLY4032 and HLY4039 (Ura ${ }^{-}$strains for hdal/hdal and $r p d 3 / r p d 3$ mutants). $\mathrm{Ura}^{+}$strains of TS3.3 [85] and HLY4032 were obtained by transforming AscI digested pBES1 16 [37], which were used for hyphal induction. hda1/hda1 yng2/yng2 mutants were generated by deleting $1 \mathcal{N} G 2$ in HLY4032 [50]. The disruption was confirmed by southern blotting (unpublished data).

A 0.9-kb PCR product (primers 1 and 2) containing the C-terminal NRG1 coding region and a 1.3-kb PCR product (primers 9 and 10) containing the $\mathrm{C}$-terminal RPD3 coding region were inserted into the BamHI-MluI sites of pPR673 [50]. The resulting plasmids were digested with $S a c I$ to target integration into their own loci to express Nrg1-13Myc and Rpd3-13Myc. A 1.3-kb PCR product (primers 5 and 6) containing the $\mathrm{C}$-terminal HDA1 coding region and a $0.8-\mathrm{kb}$ PCR product (primers 7 and 8) containing the C-terminal $1 \mathcal{N G} 2$ coding region were digested with $B g I I \mathrm{I}$ and $M l u \mathrm{I}$ and cloned into the BamHI-MluI sites of pPR673. The resulting plasmids were digested with $B a m H I$ to target the integration of the plasmid into its own locus to express Hdal-13Myc and Yng2-13Myc. The pMAL2-NRG1$13 \mathrm{Myc}$ plasmid was constructed by amplifying NRG1-13MYC (primers 3 and 4) from pACT1-NRG1-13MYG plasmid (our unpublished data). The PCR product was digested with $X b a \mathrm{I}$ and $K p n I$ to replace WOR1-HA from pMAL2-WOR1-HA plasmid [86]. The resulting plasmids were digested with $A s c \mathrm{I}$ to target integration into the ADE2 locus to express Nrg1-13Myc.

The $Y \mathcal{N} G 2$ coding sequence was amplified using primers 13 and 14. The resulting 0.9 -kb PCR product was digested with $\mathcal{N} o t \mathrm{I}$ and $M l u \mathrm{I}$ and inserted into the $\mathcal{N} o t \mathrm{I}-M l u \mathrm{I}$ site of pPR673 to create pACT1-YNG2-13MYG. A 1.6-kb 1 NG2 promoter fragment upstream of the START site of $Y \mathcal{N} G 2$ (from -1 to $-1,600$ ) was PCR amplified using primers 11 and 12. The resulting purified PGR product was digested with $S t u \mathrm{I}$ and $\mathrm{BamHI}$ and cloned into the StuI-BamHI site of pACT1-YNG2-13MYC, displacing the 1-kb ACT1 promoter region to generate pYNG2-YNG2-13MYC. Twostep PCR was used to create pYNG2-YNG2 ${ }^{\mathrm{K} 175 \mathrm{R}}-13 \mathrm{MYC}$. Two pairs of primers (primers 13 and 15, 16 and 14) were used to PCR amplify overlapping $1 \mathcal{N} G 2$ fragments with the mutation in the overlapping region. The resulting PCR products were purified and mixed as templates for another round of PCR amplification using the primers 13 and 14, which produced the full-length $r N G 2^{K 175 R}$ sequence. The resulting mutant, $Y N G 2^{K 175 R}$, was cloned into the NotI-MluI site of the plasmid pYNG2-YNG2-13MYG, replacing the wild-type copy, and was confirmed by DNA sequencing. Twostep PCR was used to create pYNG2-YNG2 ${ }^{\mathrm{K} 175 \mathrm{Q}_{-}}-13 \mathrm{MYC}$. Two pairs of primers (primers 13 and 17, and 18 and 14) were used to PCR amplify overlapping $r \mathcal{N} G 2$ fragments with the mutation in the overlapping region. Subsequent steps were done by using the same methods as pYNG2-YNG2 ${ }^{\mathrm{K} 175 \mathrm{R}}{ }_{-13 \mathrm{MYC} \text {. These plasmids }}$ were digested with the $P m I I$ within the $1 \mathcal{N} G 2$ promoter region for integration into the endogenous $Y \mathcal{N} G 2$ locus in yng2/yng2 and hda1/hda1 yng2/yng2 mutant strains.

\section{Northern and Quantitative RT-PCR Expression Analysis}

Methods for RNA isolation and Northern blot hybridization were carried out as previously described [87]. Probes for HWP1, ALS3, ECE1, and ACT1 were made by PCR amplification of 500-bp fragments from coding regions of each gene from SC5314 genomic DNA. The primers used were as follows: HWP1, primers 19 and 20; ALS3, primers 21 and 22; ECE1, primers 23 and 24; and $A C T 1$, primers 25 and 26. For quantitative real-time reverse transcription-PGR (qRT -PGR) analysis, $10 \mu \mathrm{g}$ of total RNA was DNase-treated at $37^{\circ} \mathrm{C}$ for $1 \mathrm{~h}$ using the DNA-free kit (Qiagen), cDNA was synthesized using the SuperScript II Reverse Transcriptase kit (Invitrogen), and qPCR was done using the iQ SYBR Green Supermix (Bio-Rad) using the primers 27 and 28 for $H W P 1$, primers 29 and 30 for ALS3, primers 31 and 32 for ECE1, primers 33 and 34 for ACT1, and primers 35 and 36 for NRG1. The iCycler iQ detection system (Bio-Rad) was used with the following program: initial denaturation at $94^{\circ} \mathrm{C}$ for $5 \mathrm{~min}$, followed by 40 cycles of $94^{\circ} \mathrm{C}$ for $20 \mathrm{~s}, 56^{\circ} \mathrm{C}$ for $30 \mathrm{~s}$, and $68^{\circ} \mathrm{C}$ for $20 \mathrm{~s}$. Amplification specificity was determined by melting curve analysis.

\section{Chromatin Immunoprecipitation}

Chromatin immunoprecipitation was performed as described with modifications [50,57]. Cells were formaldehyde cross-linked by adding formaldehyde $(37 \%)$ to a $1 \%$ final concentration. Treated cultures were mixed by shaking and incubated for $15 \mathrm{~min}$ at room temperature. $2.5 \mathrm{M}$ glycine was added to a final concentration of $125 \mathrm{mM}$, and treated cultures were mixed and incubated for $5 \mathrm{~min}$ at room temperature. Cells were pelleted at $3,000 \mathrm{~g}$ for $5 \mathrm{~min}$ at $4^{\circ} \mathrm{C}$, washed four times with $20 \mathrm{ml} \mathrm{PBS}$, and resuspended in $400 \mu \mathrm{l}$ of $4^{\circ} \mathrm{C}$ lysis buffer (50 mM HEPES-KOH [pH 7.5],140 mM NaCl, 1 mM EDTA, $1 \%$ Triton X-100, 0.1\% sodium deoxycholate) with protease inhibitors. All subsequent 
ChIP and wash steps were done at $4^{\circ} \mathrm{C}$. Cells were lysed using a Fast-Prep system (FP120; Thermo Electron, Waltham, MA). DNA was sheared by sonication six times for $20 \mathrm{~s}$ at high power on a Bioruptor (diagenode) with $40 \mathrm{~s}$ intervals on ice.

For the IP, $10 \mu \mathrm{l}$ of anti-Myc (SC-789, Santa Cruz), $4 \mu \mathrm{l}$ of antiH3 (ab1791; Abcam) or anti-acetylated-H4 (06-866; Millipore) antibodies were used for $\sim 4 \mathrm{mg}$ of chromatin proteins in an immunoprecipitation volume of $200 \mu \mathrm{l}$. The IP was incubated O/ $\mathrm{N}$ at $4^{\circ} \mathrm{C}$, with agitation. Then $50 \mu \mathrm{l}$ of a $50 \%$ suspension of protein A-Sepharose beads (GE Healthcare, 17-0974) in lysis buffer was added to the IP and incubated $2 \mathrm{~h}$ at $4^{\circ} \mathrm{C}$ with agitation. The beads were pelleted for $1 \mathrm{~min}$ at 3,000 g. After removal of the supernatant, the beads were washed with a series of buffers for 5 min each wash: twice in lysis buffer, twice in high-salt lysis buffer (50 mM HEPES-KOH [pH 7.5], $500 \mathrm{mM} \mathrm{NaCl}$, $1 \mathrm{mM}$ EDTA, $1 \%$ Triton X-100, 0.1\% sodium deoxycholate), twice in wash buffer $(10 \mathrm{mM}$ Tris-HCl [pH 8.0], $250 \mathrm{mM} \mathrm{LiCl,}$ $0.5 \%$ NP-40, 0.5\% sodium deoxycholate, $1 \mathrm{mM} \mathrm{EDTA),} \mathrm{and}$ once in TE (10 mM Tris, $1 \mathrm{mM}$ EDTA [pH 8.0]). After the last wash, $75 \mu$ of elution buffer (50 mM Tris-HCl [pH 8.0], $10 \mathrm{mM}$ EDTA, 1\% SDS) was added to each sample, and the beads were incubated at $65^{\circ} \mathrm{C}$ for $10 \mathrm{~min}$. The beads were spun for $1 \mathrm{~min}$ at $5000 \mathrm{~g}$, and the supernatant was removed and retained. A second elution was carried out with $75 \mu \mathrm{l}$ of elution buffer 2 (TE, $1 \%$ SDS) and eluates from the two elution steps were combined. For the ChIP input material set aside, $140 \mu$ l elution buffer 2 (TE, $1 \%$ SDS) was added to $10 \mu \mathrm{l} \mathrm{WCE.} \mathrm{ChIP} \mathrm{and} \mathrm{input} \mathrm{samples} \mathrm{were}$ incubated overnight at $65^{\circ} \mathrm{C}$ to reverse the formaldehyde crosslinks. $150 \mu \mathrm{l}$ of proteinase $\mathrm{K}$ solution (TE, $60 \mu \mathrm{g} / \mathrm{ml}$ glycogen, $500 \mu \mathrm{g} / \mathrm{ml}$ proteinase $\mathrm{K}$ ) was added to each sample, and samples were incubated at $37^{\circ} \mathrm{C}$ for $2 \mathrm{~h}$. Samples were extracted twice with $400 \mu \mathrm{l}$ Tris buffer-saturated phenol/chloroform/isoamyl alcohol solution (25:24:1). $15 \mu \mathrm{l}$ of $5 \mathrm{M} \mathrm{NaCl}$ and $0.8 \mathrm{ml}$ of $100 \%$ ethanol $\left(4^{\circ} \mathrm{C}\right)$ were added and the DNA was precipitated for $20 \mathrm{~min}$ at $-20^{\circ} \mathrm{C}$, then pelleted by centrifugation at $14,000 \mathrm{~g}$ for $10 \mathrm{~min}$ at $4^{\circ} \mathrm{C}$, washed once with cold $70 \%$ ethanol, and allowed to air dry. The samples were resuspended in $30 \mu \mathrm{l}$ of TE containing $100 \mu \mathrm{g} /$ $\mathrm{ml}$ RNaseA and incubated for $1 \mathrm{~h}$ at $37^{\circ} \mathrm{C}$. DNA derived from the whole cell extracts and immunoprecipitation (IP) eluate was analyzed by quantitative PCR (qPCR). Plotted are the average and standard error of the mean of three independent qPCR reactions for each experiment.

\section{Immunoprecipitation}

Acetylated Yng2-Myc was immunoprecipitated with $30 \mu \mathrm{l}$ protein A-sepharose beads (GE Healthcare, 17-0974) conjugated with $10 \mu \mathrm{l}$ of rabbit polyclonal anti-acetylated-lysine (Cell Signaling, 9441S) at $4^{\circ} \mathrm{C}$ overnight in $200 \mu \mathrm{l}$ pre-cleared WCEs, and detected with a peroxidase-conjugated anti-c-Myc antibody (Roche).

\section{Supporting Information}

Figure S1 Nrgl is detected in the nucleus of both apical and subapical cells of hyphae. Wild type cells carrying Nrgl-Myc (HLY3922) were processed for indirect immunofluorescence, as described [88]. Cells were grown in YPD+10\% Serum medium at $37^{\circ} \mathrm{C}$ for hyphal induction or in YPD medium for yeast growth. Cells were fixed at $5 \mathrm{~h}$ after induction and stained for Nrg1-Myc with 9E10 mouse antibodies (Covance) and FITC-conjugated secondary antibodies (Jackson Laboratory). DNA was stained with DAPI. An untagged control (SC5314) was included.

(TIF)
Figure S2 Promoter recruitment of $\mathrm{Hdal}$ is required for hyphal maintenance by inhibiting $\mathrm{Nrg} 1$ access to the promoters of hypha-specific genes. (A) $A L S 3$ and ECE1 mRNA levels were determined by qRT-PCR as described in Figure 3 (B) Kinetics of Nrgl-Myc (B) and Hdal-Myc $(\mathrm{C})$ binding at the ALS3 and ECE1 promoters were determined by ChIP as described in Figure 3C,D.

(TIF)

Figure S3 Rpd3-Myc disassociates rapidly from the promoters of hypha-specific genes upon hyphal induction, determined by ChIP as described in Figure 1B.

(TIF)

Figure S4 The function of Hdal in sustained hyphal transcription is mediated through Yng2 deacetylation (A) ALS3 and ECE1 mRNA levels were determined by qRT-PCR as described in Figure 4(B) Relative Yng2 enrichment (B), relative H3 occupancy (C), and $\mathrm{H} 4$ acetylation level (D) at ALS3 and ECE1 promoter. ChIPs were performed as described in Figure 4E,F,G.

(TIF)

Figure S5 Kinetics of Yng2-Myc and Yng2 ${ }^{\mathrm{K} 175 \mathrm{R}}-\mathrm{Myc}$ promoter binding in hda1 mutants by ChIP with anti-Myc as described in Figure 5C.

(TIF)

Table S1 Yng2 deacetylation by Hda1 is not required for germ tube formation. (A) Germ tube formation of wild type and hda1/ hda1. Cells of wild type (TS3.3+pBES116) and hda1/hda1 (HLY4032+pBES116) were diluted 1:100 into indicated medium at $37^{\circ} \mathrm{C}$. The percentage of cells forming germ tubes in YPD $+10 \%$ serum medium, Spider medium, and M199 pH 8 medium at $60 \mathrm{~min}$ or in Lee's medium at $180 \mathrm{~min}$ was determined by counting at least 300 cells/sample, in triplicate. The samples from Spider medium were gently sonicated to disrupt clumping. Mean (\% germ tube formation) $\pm \mathrm{SE}$ (standard error) of two independent experiments. The hdal/hdal mutant is able to form germ tube in YPD with serum and Spider media but shows a dramatically reduced level of germ tube formation in M199 and Lee's media. This is likely due to impaired growth of the mutant in the media. The doubling time of the wild type (TS3.3+pBES116) and hda1/hda1 (HLY4032+pBES116) in YPD medium at $30^{\circ} \mathrm{C}$ is $105 \mathrm{~min}$ and $135 \mathrm{~min}$, respectively, and in M199 PH 8 medium at $30^{\circ} \mathrm{C}$ is $150 \mathrm{~min}$ and over $18 \mathrm{~h}$, respectively. The defect of hda1/ hda1 cells in germ tube formation in M199 is consistent with the report by Zacchi et al. [89]. (B) $y n g 2^{K 175 Q}$ mutant has no dramatic defect in germ tube formation. Cells of wild type $Y \mathcal{N} G 2$ (HLY4035), $y n g 2^{\text {K175R }}$ (HLY4036), and $y n g 2^{\text {K175Q }}$ (HLY4037) were diluted 1:100 into indicated medium at $37^{\circ} \mathrm{C}$. The percentage of cells forming germ tubes was calculated as described in (A). The two $y n g 2$ mutants show a similar growth rate as the $1 \mathcal{N} G 2$ strain in all media examined.

(PDF)

\section{Acknowledgments}

We thank Drs. D. Soll, J.Y. Chen, M. Whiteway, A. Mitchell, and J. Ernst for C. albicans strains, and G.R. Fink for comments on the manuscript.

\section{Author Contributions}

Conceived and designed the experiments: YL AW HL. Performed the experiments: YL CS AW. Analyzed the data: YL CS HL. Wrote the paper: YL HL. 


\section{References}

1. Odds FC (1988) Candida and candidosis. Philadelphia, PA: Bailliere Tindall.

2. Kumamoto CA, Vinces MD (2005) Contributions of hyphae and hypha-coregulated genes to Candida albicans virulence. Cell Microbiol 7: 1546-1554.

3. Staab JF, Bradway SD, Fidel PL, Sundstrom P (1999) Adhesive and mammalian transglutaminase substrate properties of Candida albicans Hwp1. Science 283: 1535-1538.

4. Almeida RS, Brunke S, Albrecht A, Thewes S, Laue M, et al. (2008) The hyphal-associated adhesin and invasin Als3 of Candida albicans mediates iron acquisition from host ferritin. PLoS Pathog 4: e1000217. doi:10.1371/journal .ppat.1000217.

5. Phan QT, Myers CL, Fu Y, Sheppard DC, Yeaman MR, et al. (2007) Als3 is a Candida albicans invasin that binds to cadherins and induces endocytosis by host cells. PLoS Biol 5: e64. doi:10.1371/journal.pbio.0050064.

6. Weissman Z, Kornitzer D (2004) A family of Candida cell surface haem-binding proteins involved in haemin and haemoglobin-iron utilization. Mol Microbiol 53: $1209-1220$.

7. Hogan DA, Sundstrom P (2009) The Ras/cAMP/PKA signaling pathway and virulence in Candida albicans. Future Microbiol 4: 1263-1270.

8. Rocha CR, Schroppel K, Harcus D, Marcil A, Dignard D, et al. (2001) Signaling through adenylyl cyclase is essential for hyphal growth and virulence in the pathogenic fungus Candida albicans. Mol Biol Cell 12: 3631-3643.

9. Bahn YS, Sundstrom P (2001) CAP1, an adenylate cyclase-associated protein gene, regulates bud-hypha transitions, filamentous growth, and cyclic AMP levels and is required for virulence of Candida albicans. J Bacteriol 183: 3211-3223.

10. Zou H, Fang HM, Zhu Y, Wang Y (2009) Candida albicans Cyr1, Capl and Gactin form a sensor/effector apparatus for activating cAMP synthesis in hyphal growth. Mol Microbiol.

11. Cassola A, Parrot M, Silberstein S, Magee BB, Passeron S, et al. (2004) Candida albicans lacking the gene encoding the regulatory subunit of protein kinase A displays a defect in hyphal formation and an altered localization of the catalytic subunit. Eukaryot Cell 3: 190-199.

12. Bockmuhl DP, Krishnamurthy S, Gerads M, Sonneborn A, Ernst JF (2001) Distinct and redundant roles of the two protein kinase A isoforms Tpklp and Tpk2p in morphogenesis and growth of Candida albicans. Mol Microbiol 42: 1243-1257.

13. Sonneborn A, Bockmuhl DP, Gerads M, Kurpanek K, Sanglard D, et al. (2000) Protein kinase A encoded by TPK 2 regulates dimorphism of Candida albicans. Mol Microbiol 35: 386-396.

14. Lo HJ, Kohler JR, DiDomenico B, Loebenberg D, Cacciapuoti A, et al. (1997) Nonfilamentous C. albicans mutants are avirulent. Cell 90: 939-949.

15. Stoldt VR, Sonneborn A, Leuker CE, Ernst JF (1997) Efglp, an essential regulator of morphogenesis of the human pathogen Candida albicans, is a member of a conserved class of bHLH proteins regulating morphogenetic processes in fungi. Embo J 16: 1982-1991.

16. Cao F, Lane S, Raniga PP, Lu Y, Zhou Z, et al. (2006) The Flo8 transcription factor is essential for hyphal development and virulence in Candida albicans. Mol Biol Cell 17: 295-307.

17. Bockmuhl DP, Ernst JF (2001) A potential phosphorylation site for an A-type kinase in the Efgl regulator protein contributes to hyphal morphogenesis of Candida albicans. Genetics 157: 1523-1530.

18. Harcus D, Nantel A, Marcil A, Rigby T, Whiteway M (2004) Transcription profiling of cyclic AMP signaling in Candida albicans. Mol Biol Cell 15: 4490-4499.

19. Braun BR, Johnson AD (1997) Control of filament formation in Candida albicans by the transcriptional repressor TUP1. Science 277: 105-109.

20. Garcia-Sanchez S, Mavor AL, Russell CL, Argimon S, Dennison P, et al. (2005) Global roles of Ssn6 in Tup 1- and Nrg1-dependent gene regulation in the fungal pathogen, Candida albicans. Mol Biol Cell 16: 2913-2925.

21. Hwang CS, Oh JH, Huh WK, Yim HS, Kang SO (2003) Ssn6, an important factor of morphological conversion and virulence in Candida albicans. Mol Microbiol 47: 1029-1043.

22. Kadosh D, Johnson AD (2005) Induction of the Candida albicans filamentous growth program by relief of transcriptional repression: a genome-wide analysis. Mol Biol Cell 16: 2903-2912.

23. Khalaf RA, Zitomer RS (2001) The DNA binding protein Rfgl is a repressor of filamentation in Candida albicans. Genetics 157: 1503-1512.

24. Murad AM, d'Enfert C, Gaillardin C, Tournu H, Tekaia F, et al. (2001) Transcript profiling in Candida albicans reveals new cellular functions for the transcriptional repressors CaTup1, CaMig1 and CaNrg1. Mol Microbiol 42: 981-993.

25. Murad AM, Leng P, Straffon M, Wishart J, Macaskill S, et al. (2001) NRG1 represses yeast-hypha morphogenesis and hypha-specific gene expression in Candida albicans. Embo J 20: 4742-4752.

26. Braun BR, Kadosh D, Johnson AD (2001) NRG1, a repressor of filamentous growth in C.albicans, is down-regulated during filament induction. Embo J 20: 4753-4761.

27. Bauer J, Wendland J (2007) Candida albicans Sfl1 suppresses flocculation and filamentation. Eukaryot Cell 6: 1736-1744.

28. Park YN, Morschhauser J (2005) Tetracycline-inducible gene expression and gene deletion in Candida albicans. Eukaryot Cell 4: 1328-1342.
29. Saville SP, Lazzell AL, Bryant AP, Fretzen A, Monreal A, et al. (2006) Inhibition of filamentation can be used to treat disseminated candidiasis. Antimicrob Agents Chemother 50: 3312-3316.

30. Enjalbert B, Whiteway M (2005) Release from quorum-sensing molecules triggers hyphal formation during Candida albicans resumption of growth. Eukaryot Cell 4: 1203-1210.

31. Klengel T, Liang WJ, Chaloupka J, Ruoff C, Schroppel K, et al. (2005) Fungal adenylyl cyclase integrates $\mathrm{CO} 2$ sensing with cAMP signaling and virulence. Curr Biol 15: 2021-2026.

32. Xu XL, Lee RT, Fang HM, Wang YM, Li R, et al. (2008) Bacterial peptidoglycan triggers Candida albicans hyphal growth by directly activating the adenylyl cyclase Cyrlp. Cell Host Microbe 4: 28-39.

33. Shapiro RS, Uppuluri P, Zaas AK, Collins C, Senn H, et al. (2009) Hsp90 orchestrates temperature-dependent Candida albicans morphogenesis via Ras 1PKA signaling. Curr Biol 19: 621-629.

34. Maidan MM, De Rop L, Serneels J, Exler S, Rupp S, et al. (2005) The G protein-coupled receptor Gpr1 and the G\{alpha\} protein Gpa2 act through the cAMP-protein kinase A pathway to induce morphogenesis in candida albicans. Mol Biol Cell 16: 1971-1986.

35. Miwa T, Takagi Y, Shinozaki M, Yun CW, Schell WA, et al. (2004) Gprl, a putative G-protein-coupled receptor, regulates morphogenesis and hypha formation in the pathogenic fungus Candida albicans. Eukaryot Cell 3: 919-931.

36. Sanchez-Martinez C, Perez-Martin J (2002) Gpa2, a G-protein alpha subunit required for hyphal development in Candida albicans. Eukaryot Cell 1: 865-874.

37. Feng Q, Summers E, Guo B, Fink G (1999) Ras signaling is required for seruminduced hyphal differentiation in Candida albicans. J Bacteriol 181: 6339-6346.

38. Zhu Y, Fang HM, Wang YM, Zeng GS, Zheng XD, et al. (2009) Ras1 and Ras2 play antagonistic roles in regulating cellular cAMP level, stationary-phase entry and stress response in Candida albicans. Mol Microbiol 74: 862-875.

39. Hornby JM, Jensen EC, Lisec AD, Tasto JJ, Jahnke B, et al. (2001) Quorum sensing in the dimorphic fungus Candida albicans is mediated by farnesol. Appl Environ Microbiol 67: 2982-2992.

40. Davis-Hanna A, Piispanen AE, Stateva LI, Hogan DA (2008) Farnesol and dodecanol effects on the Candida albicans Ras1-cAMP signalling pathway and the regulation of morphogenesis. Mol Microbiol 67: 47-62.

41. Bastidas RJ, Heitman J, Cardenas ME (2009) The protein kinase Tor1 regulates adhesin gene expression in Candida albicans. PLoS Pathog 5: e1000294. doi:10.1371/journal.ppat. 1000294.

42. Cutler NS, Pan X, Heitman J, Cardenas ME (2001) The TOR sional transduction cascade controls cellular differentiation in response to nutrients. Mol Biol Cell 12: 4103-4113.

43. Peterson CL, Laniel MA (2004) Histones and histone modifications. Curr Biol 14: R546-R551.

44. Cairns BR (2005) Chromatin remodeling complexes: strength in diversity, precision through specialization. Curr Opin Genet Dev 15: 185-190.

45. Sarma K, Reinberg D (2005) Histone variants meet their match. Nat Rev Mol Cell Biol 6: 139-149.

46. Adkins MW, Howar SR, Tyler JK (2004) Chromatin disassembly mediated by the histone chaperone Asfl is essential for transcriptional activation of the yeast PHO5 and PHO8 genes. Mol Cell 14: 657-666.

47. Adkins MW, Tyler JK (2006) Transcriptional activators are dispensable for transcription in the absence of Spt6-mediated chromatin reassembly of promoter regions. Mol Cell 21: 405-416.

48. Millar CB, Grunstein M (2006) Genome-wide patterns of histone modifications in yeast. Nat Rev Mol Cell Biol 7: 657-666.

49. Lee KK, Workman JL (2007) Histone acetyltransferase complexes: one size doesn't fit all. Nat Rev Mol Cell Biol 8: 284-295.

50. Lu Y, Su C, Mao X, Raniga PP, Liu H, et al. (2008) Efgl-mediated recruitment of NuA4 to promoters is required for hypha-specific Swi/Snf binding and activation in Candida albicans. Mol Biol Cell 19: 4260-4272.

51. Saville SP, Lazzell AL, Monteagudo C, Lopez-Ribot JL (2003) Engineered control of cell morphology in vivo reveals distinct roles for yeast and filamentous forms of Candida albicans during infection. Eukaryot Cell 2: 1053-1060.

52. Homann OR, Dea J, Noble SM, Johnson AD (2009) A phenotypic profile of the Candida albicans regulatory network. PLoS Genet 5: e1000783. doi:10.1371/ journal.pgen.1000783.

53. Banerjee M, Thompson DS, Lazzell A, Carlisle PL, Pierce C, et al. (2008) UME6, a novel filament-specific regulator of Candida albicans hyphal extension and virulence. Mol Biol Cell 19: 1354-1365.

54. Srikantha T, Tsai L, Daniels K, Klar AJ, Soll DR (2001) The histone deacetylase genes HDA1 and RPD3 play distinct roles in regulation of high-frequency phenotypic switching in Candida albicans. J Bacteriol 183: 4614-4625.

55. Wu J, Suka N, Carlson M, Grunstein M (2001) TUP1 utilizes histone H3/H2Bspecific HDA1 deacetylase to repress gene activity in yeast. Mol Cell 7: 117-126.

56. Lin YY, Qi Y, Lu JY, Pan X, Yuan DS, et al. (2008) A comprehensive synthetic genetic interaction network governing yeast histone acetylation and deacetylation. Genes Dev 22: 2062-2074.

57. Pokholok DK, Harbison CT, Levine S, Cole M, Hannett NM, et al. (2005) Genome-wide map of nucleosome acetylation and methylation in yeast. Cell 122: $517-527$. 
58. Lee KL, Buckley HR, Campbell CC (1975) An amino acid liquid synthetic medium for the development of mycelial and yeast forms of Candida Albicans. Sabouraudia 13: 148-153.

59. Wullschleger S, Loewith R, Hall MN (2006) TOR signaling in growth and metabolism. Cell 124: 471-484.

60. Zacchi LF, Gomez-Raja J, Davis DA (2010) Mds3 regulates morphogenesis in Candida albicans through the TOR pathway. Mol Cell Biol 30: 3695-3710.

61. Kucharski R, Maleszka J, Foret S, Maleszka R (2008) Nutritional control of reproductive status in honeybees via DNA methylation. Science 319: $1827-1830$.

62. Araki K, Turner AP, Shaffer VO, Gangappa S, Keller SA, et al. (2009) mTOR regulates memory CD8 T-cell differentiation. Nature 460: 108-112.

63. Yilmaz OH, Valdez R, Theisen BK, Guo W, Ferguson DO, et al. (2006) Pten dependence distinguishes haematopoietic stem cells from leukemia-initiating cells. Nature 441: 475-482.

64. Hobbs RM, Seandel M, Falciatori I, Rafii S, Pandolfi PP (2010) Plzf regulates germline progenitor self-renewal by opposing mTORC1. Cell 142: 468-479.

65. Castilho RM, Squarize CH, Chodosh LA, Williams BO, Gutkind JS (2009) mTOR mediates Wnt-induced epidermal stem cell exhaustion and aging. Cell Stem Cell 5: 279-289.

66. Braun BR, Johnson AD (2000) TUP1, CPH1 and EFG1 make independent contributions to filamentation in candida albicans. Genetics 155: 57-67.

67. Kebaara BW, Langford ML, Navarathna DH, Dumitru R, Nickerson KW, et al. (2008) Candida albicans Tupl is involved in farnesol-mediated inhibition of filamentous-growth induction. Eukaryot Cell 7: 980-987.

68. Cruz MC, Goldstein AL, Blankenship J, Del Poeta M, Perfect JR, et al. (2001) Rapamycin and less immunosuppressive analogs are toxic to Candida albicans and Cryptococcus neoformans via FKBP12-dependent inhibition of TOR. Antimicrob Agents Chemother 45: 3162-3170.

69. Crespo JL, Powers T, Fowler B, Hall MN (2002) The TOR-controlled transcription activators GLN3, RTG1, and RTG3 are regulated in response to intracellular levels of glutamine. Proc Natl Acad Sci U S A 99: 6784-6789.

70. Carlisle PL, Banerjee M, Lazzell A, Monteagudo C, Lopez-Ribot JL, et al. (2009) Expression levels of a filament-specific transcriptional regulator are sufficient to determine Candida albicans morphology and virulence. Proc Natl Acad Sci U S A 106: 599-604.

71. Zeidler U, Lettner T, Lassnig C, Muller M, Lajko R, et al. (2009) UME6 is a crucial downstream target of other transcriptional regulators of true hyphal development in Candida albicans. FEMS Yeast Res 9: 126-142.

72. Zheng X, Wang Y (2004) Hgcl, a novel hypha-specific G1 cyclin-related protein regulates Candida albicans hyphal morphogenesis. Embo J 23: 1845-1856.

73. Sinha I, Wang YM, Philp R, Li CR, Yap WH, et al. (2007) Cyclin-dependent kinases control septin phosphorylation in Candida albicans hyphal development. Dev Cell 13: 421-432.

74. Zheng XD, Lee RT, Wang YM, Lin QS, Wang Y (2007) Phosphorylation of Rga2, a Cdc42 GAP, by CDK/Hgc1 is crucial for Candida albicans hyphal growth. EMBO J 26: 3760-3769.
75. Wang A, Raniga PP, Lane S, Lu Y, Liu H (2009) Hyphal chain formation in Candida albicans: Cdc28-Hgcl phosphorylation of Efgl represses cell separation genes. Mol Cell Biol 29: 4406-4416.

76. Bishop A, Lane R, Beniston R, Chapa-y-Lazo B, Smythe C, et al. (2010) Hyphal growth in Candida albicans requires the phosphorylation of Sec2 by the Cdc28Ccn1/Hgcl kinase. EMBO J 29: 2930-2942.

77. Uppuluri P, Chaturvedi AK, Srinivasan A, Banerjee M, Ramasubramaniam AK, et al. (2010) Dispersion as an important step in the Candida albicans biofilm developmental cycle. PLoS Pathog 6: e1000828. doi:10.1371/journal.ppat.1000828.

78. Pappas PG, Alexander BD, Andes DR, Hadley S, Kauffman CA, et al. (2010) Invasive fungal infections among organ transplant recipients: results of the Transplant-Associated Infection Surveillance Network (TRANSNET). Clin Infect Dis 50: 1101-1111.

79. De Nadal E, Zapater M, Alepuz PM, Sumoy L, Mas G, et al. (2004) The MAPK Hog1 recruits Rpd3 histone deacetylase to activate osmoresponsive genes. Nature 427: 370-374.

80. Sertil O, Vemula A, Salmon SL, Morse RH, Lowry CV (2007) Direct role for the Rpd3 complex in transcriptional induction of the anaerobic DAN/TIR genes in yeast. Mol Cell Biol 27: 2037-2047.

81. Sharma VM, Tomar RS, Dempsey AE, Reese JC (2007) Histone deacetylases RPD3 and HOS2 regulate the transcriptional activation of DNA damageinducible genes. Mol Cell Biol 27: 3199-3210.

82. Wang A, Kurdistani SK, Grunstein M (2002) Requirement of Hos2 histone deacetylase for gene activity in yeast. Science 298: 1412-1414.

83. Wang Z, Zang C, Cui K, Schones DE, Barski A, et al. (2009) Genome-wide mapping of HATs and HDACs reveals distinct functions in active and inactive genes. Cell 138: 1019-1031.

84. Liu H, Kohler J, Fink GR (1994) Suppression of hyphal formation in Candida albicans by mutation of a STE12 homolog. Science 266: 1723-1726.

85. Srikantha T, Tsai L, Daniels K, Enger L, Highley K, et al. (1998) The twocomponent hybrid kinase regulator CaNIK1 of Candida albicans. Microbiology 144(Pt 10): 2715-2729.

86. Huang G, Wang H, Chou S, Nie X, Chen J, et al. (2006) Bistable expression of WOR1, a master regulator of white-opaque switching in Candida albicans. Proc Natl Acad Sci U S A 103: 12813-12818.

87. Lane S, Birse C, Zhou S, Matson R, Liu H (2001) DNA array studies demonstrate convergent regulation of virulence factors by Cph1, Cph2, and Efg1 in Candida albicans. J Biol Chem 276: 48988-48996.

88. Wang A, Lane S, Tian Z, Sharon A, Hazan I, et al. (2007) Temporal and spatial control of HGC1 expression results in Hgcl localization to the apical cells of hyphae in Candida albicans. Eukaryot Cell 6: 253-261.

89. Zacchi LF, Schulz WL, Davis DA (2010) HOS2 and HDAl encode histone deacetylases with opposing roles in Candida albicans morphogenesis. PLoS One 5: e12171. doi:10.1371/journal.pone.0012171. 